Article

\title{
Neutrosophic Triangular Norms and Their Derived Residuated Lattices
}

\author{
Qingqing Hu and Xiaohong Zhang * \\ School of Arts and Sciences, Shaanxi University of Science \& Technology, Xi'an 710021, China; \\ huqingqing122@163.com \\ * Correspondence: zhangxiaohong@sust.edu.cn or zxhonghz@263.net
}

Received: 21 May 2019; Accepted: 14 June 2019; Published: 20 June 2019

\begin{abstract}
Neutrosophic triangular norms (t-norms) and their residuated lattices are not only the main research object of neutrosophic set theory, but also the core content of neutrosophic logic. Neutrosophic implications are important operators of neutrosophic logic. Neutrosophic residual implications based on neutrosophic t-norms can be applied to the fields of neutrosophic inference and neutrosophic control. In this paper, neutrosophic t-norms, neutrosophic residual implications, and the residuated lattices derived from neutrosophic t-norms are investigated deeply. First of all, the lattice and its corresponding system are proved to be a complete lattice and a De Morgan algebra, respectively. Second, the notions of neutrosophic t-norms are introduced on the complete lattice discussed earlier. The basic concepts and typical examples of representable and non-representable neutrosophic t-norms are obtained. Naturally, De Morgan neutrosophic triples are defined for the duality of neutrosophic $\mathrm{t}$-norms and neutrosophic $\mathrm{t}$-conorms with respect to neutrosophic negators. Third, neutrosophic residual implications generated from neutrosophic t-norms and their basic properties are investigated. Furthermore, residual neutrosophic t-norms are proved to be infinitely $\checkmark$-distributive, and then some important properties possessed by neutrosophic residual implications are given. Finally, a method for producing neutrosophic t-norms from neutrosophic implications is presented, and the residuated lattices are constructed on the basis of neutrosophic t-norms and neutrosophic residual implications.
\end{abstract}

Keywords: neutrosophic sets; neutrosophic triangular norms; residuated lattices; representable neutrosophic t-norms; De Morgan neutrosophic triples; neutrosophic residual implications; infinitely $\vee$-distributive

\section{Introduction}

Neutrosophic sets were firstly proposed by Smarandache [1] from a philosophical point of view in 1998, which is a generalization of fuzzy sets and intuitionistic fuzzy sets. However, it is difficult to apply neutrosophic sets to solve practical problems since the values of their three functions with respect to truth, indeterminacy and falsity lie in $] 0-, 1+[$. The definition of single-valued neutrosophic sets were introduced by Wang [2], whose values belong to [0,1]. With the development of neutrosophic set theory, single-valued neutrosophic sets and their applications have been investigated by more scholars. Single-valued neutrosophic sets were successfully applied to various decision making problems [3-8]. In addition, Zhang et al. studied the neutrosophic logic algebras and discussed neutrosophic filters and neutrosophic triplet groups, which are the important foundation of the development of neutrosophic logic theory [9-12]. To facilitate research, "single-valued neutrosophic sets" are abbreviated as "neutrosophic sets" in this paper. For neutrosophic sets, the truth-membership, indeterminacy-membership and falsity-membership are not restricted to each other, which is different from intuitionistic fuzzy sets. Picture fuzzy sets proposed by Cuong [13] in 2013 is a direct 
generalization of intuitionistic fuzzy sets, because their positive membership, neutral membership and negative membership are not independent completely. It is worth noting that picture fuzzy sets can be regarded as special neutrosophic sets $[14,15]$, and also can be called standard neutrosophic sets $[1,2,16-19]$.

Fuzzy logic plays a vital role in fuzzy set theory. T-norms, t-conorms, negators and implications are very important fuzzy logic operators. T-norms were originally defined by Menger [20], and then Schweizer and Sklar [21,22] redefined the t-norms which have been used to today. From the perspective of fuzzy logic, t-norms are the extension of intersection operation of fuzzy sets [23]. The algebraic properties of t-norms, for example, continuity, archimedean, strict, nilpotent and so on, are discussed in some papers [24-28]. Hu et al. studied t-norm extension operations [29]. Wang et al. discussed the lattice structure of algebra of fuzzy values [30]. The t-norms which satisfy the residual principle are an important class of t-norms, because they can produce fuzzy implications and constitute the residuated lattices [31-34]. Type-2 $t$-norms ( $t$-conorms) and their residual operators on type-2 fuzzy sets were investigated by Li [25], which promote the development of fuzzy reference system. Intuitionistic fuzzy t-norms on intuitionistic fuzzy sets ( $L^{*}$-fuzzy sets) were proposed by Deschrijver et al., they discussed t-representable intuitionistic fuzzy t-norms and their residual operators [35,36]. Picture fuzzy sets are particular L-fuzzy sets [37]. Picture fuzzy t-norms on picture fuzzy sets were introduced in [17,38,39], some basic picture fuzzy logic connectives and their properties for picture fuzzy sets are investigated in $[40,41]$. Some classes of representable picture fuzzy t-norms and representable picture fuzzy $\mathrm{t}$-conorms on picture fuzzy sets and De Morgan picture operator triples in picture fuzzy logic are discussed [42]. Furthermore, a picture inference system is proposed by Son [43]. The residual operations, residual implications of uninorms were discussed by Baets [44]. Wang proposed the notions of residual implications (co-implications) of pseudo t-norms, left and right uninorms and studied some properties of infinitely $\vee$-distributive $(\wedge$-distributive) pseudo t-norms, left and right uninorms [45-47]. Then Liu introduced semi-uninorms and their residual implications [48].

Neutrosophic t-norms, neutrosophic t-conorms, neutrosophic negators and neutrosophic implications are important neutrosophic logic operators for neutrosophic sets. It is a very meaningful topic to discuss neutrosophic t-norms and their residual implications on neutrosophic sets. In the last few years, although Alkhazaleh discusses some neutrosophic t-norms and t-conorms in [49], Liu proposes aggregation operators based on Archimedean t-norms and t-conorms for neutrosophic numbers in [5], Smarandache discussed neutrosophic norms (n-norms), n-valued refined neutrosophic logic and its applications in physics [50,51], there are a few papers about basic neutrosophic logic connectives and their properties and neutrosophic logic inference systems and their applications in the field of control. Therefore, it is necessary to study neutrosophic logic operators and their properties, especially the application of neutrosophic residual implications in neutrosophic inference and neutrosophic control.

To achieve these goals, the definitions of neutrosophic t-norms should be given firstly. We can study neutrosophic logic and neutrosophic inference systems further only if neutrosophic t-norms and their residual implications are studied thoroughly. Thus, it is the main task of this paper to study neutrosophic t-norms and their residual implications. Section 2 presents some basic notions. In Section 3, the lattice structure of neutrosophic sets is analyzed and constructed systematically based on the first type inclusion relation on neutrosophic sets. In particular, we combine some basic algebraic operations: Union, intersection and complement and their related properties to prove that the system $\left(D^{*} ; \vee_{1}, \wedge_{1}{ }^{c}, 0_{D^{*}}, 1_{D^{*}}\right)$ is a De Morgan algebra. In Section 4 , we introduce neutrosophic $\mathrm{t}$-norms (t-conorms), representable neutrosophic t-norms (t-conorms) and De Morgan neutrosophic triples. In addition, we present some important theorems and typical examples. In Section 5, the definitions of neutrosophic residual implications (co-implications) are obtained and their basic properties are discussed deeply. Moreover, residual neutrosophic t-norms (t-conorms) are proved to be infinitely $\vee$-distributive $(\wedge$-distributive), and then some important results related to residual 
neutrosophic t-norms and neutrosophic residual implications are given. Section 6 shows a method for obtaining neutrosophic t-norms from neutrosophic implications, and then proves that the system $\left(D^{*} ; \vee_{1}, \wedge_{1}, \otimes, \rightarrow, 0_{D^{*}}, 1_{D^{*}}\right)$ is a residuated lattice. In Section 7 , we conclude the paper.

\section{Preliminaries}

Some basic concepts in fuzzy set theory will be reviewed in this section.

Definition 1. ([52]) Let $U$ be a nonempty set. An intuitionistic fuzzy set $M$ in $U$ is characterized by a membership function $\mu_{M}(u)$ and a non-membership function $v_{M}(u)$. Then, an intuitionistic fuzzy set $M$ can be denoted by

$$
M=\left\{\left(u, \mu_{M}(u), v_{M}(u)\right) \mid u \in U\right\},
$$

where $\mu_{M}(u): U \rightarrow[0,1]$ and $v_{M}(u): U \rightarrow[0,1]$ with the condition $0 \leq \mu_{M}(u)+v_{M}(u) \leq 1$, for all $u \in U$. Here $\mu_{M}(u), v_{M}(u) \in[0,1]$ denote the membership and the non-membership functions of the intuitionistic fuzzy set $M$, respectively.

Definition 2. ([1]) Let $U$ be a nonempty set. A neutrosophic set $M$ in $U$ is characterized by three functions: Truth-membership function $T_{M}(u)$, indeterminacy-membership function $I_{M}(u)$, and falsity-membership function $F_{M}(u)$. Here, $\left.T_{M}(u): U \rightarrow\right]^{-} 0,1^{+}\left[, I_{M}(u): U \rightarrow\right]^{-} 0,1^{+}\left[\text {, and } F_{M}(u): U \rightarrow\right]^{-} 0,1^{+}[$with the condition $-0 \leq \sup T_{M}(u)+\sup I_{M}(u)+\sup F_{M}(u) \leq 3^{+}$, for all $u \in U$.

However, there are a lot of limitations in solving practical problems with neutrosophic sets, because their notions are given from a philosophical perspective. Thus, the concepts of single-valued neutrosophic sets are given by Wang et al. as follows.

Definition 3. ([2]) Let $U$ be a nonempty set. A single-valued neutrosophic set $M$ in $U$ is characterized by three functions: Truth-membership function $T_{M}(u)$, indeterminacy-membership function $I_{M}(u)$, and falsity-membership function $F_{M}(u)$. Then, a single-valued neutrosophic set $M$ can be denoted by

$$
M=\left\{\left\langle u, T_{M}(u), I_{M}(u), F_{M}(u)\right\rangle \mid u \in U\right\},
$$

where $T_{M}(u), I_{M}(u), F_{M}(u) \in[0,1]$ with the condition $0 \leq T_{M}(u)+I_{M}(u)+F_{M}(u) \leq 3$, for all $u \in U$.

So far, scholars have described the inclusion relation of neutrosophic sets from three different angles. The first definition is proposed by Smarandache $[1,18,53]$ and denoted as $\subseteq_{1}$; the second one is mentioned in $[2,14,54]$ and denoted by $\subseteq_{2}$; the third one is presented in $[14,38,39,54]$ and denoted by $\subseteq_{3}$. Furthermore, based on the correlation between union, intersection operations and inclusion relation, we can obtain three different types of union, intersection operations and their properties. In this paper, we consider the first type inclusion relation.

Definition 4. ([1,18,53]) Let $U$ be a nonempty set. Suppose that $M=\left\{\left\langle u, T_{M}(u), I_{M}(u), F_{M}(u)\right\rangle \mid u \in U\right\}$ and $N=\left\{\left\langle u, T_{N}(u), I_{N}(u), F_{N}(u)\right\rangle \mid u \in U\right\}$ are two neutrosophic sets in $U$. The first type inclusion relation $\subseteq_{1}$ and its basic algebraic operations are defined as follows:

(1) $M \subseteq_{1} N$ if and only if $T_{M}(u) \leq T_{M}(u), I_{M}(u) \geq I_{N}(u), F_{N}(u) \geq F_{N}(u)$, for all $u \in U$;

(2) $M \cup_{1} N=\left\{\left\langle u, \max \left(T_{M}(u), T_{N}(u)\right), \min \left(I_{M}(u), I_{N}(u)\right), \min \left(F_{M}(u), F_{N}(u)\right)\right\rangle \mid u \in U\right\}$;

(3) $M \cap_{1} N=\left\{\left\langle u, \min \left(T_{M}(u), T_{N}(u)\right), \max \left(I_{M}(u), I_{N}(u)\right), \max \left(F_{M}(u), F_{N}(u)\right)\right\rangle \mid u \in U\right\}$;

(4) $M^{c}=\left\{\left\langle u, I_{M}(u), 1-F_{M}(u), T_{M}(u)\right\rangle \mid u \in U\right\}$.

Definition 5. ([34]) Let $\left(L ; \leq_{L}\right)$ be a complete lattice. A t-norm on $\left(L ; \leq_{L}\right)$ is a commutative, associative, increasing mapping $\mathscr{T}: L^{2} \rightarrow L$, which satisfies $\mathscr{T}\left(1_{L}, u\right)=u$, for all $u \in L$.

A t-conorm on $\left(L ; \leq_{L}\right)$ is a commutative, associative, increasing mapping $\mathscr{S}: L^{2} \rightarrow L$, which satisfies $\mathscr{S}\left(0_{L}, u\right)=u$, for all $u \in L$. 
Example 1. ([27]) Some basic $t$-norms and their residual implications on $([0,1] ; \leq)$ (Table 1$)$ are defined as follows, for all $u, v \in[0,1]$,

Table 1. Some basic t-norms and their residual implications on $([0,1] ; \leq)$.

\begin{tabular}{|c|c|}
\hline t-Norms & Residual Implications \\
\hline$T_{M}(u, v)=\min (u, v)$ & $I_{G D}(u, v)= \begin{cases}1 & \text { if } u \leq v \\
v & \text { otherwise }\end{cases}$ \\
\hline$T_{P}(u, v)=u \cdot v$ & $I_{G G}(u, v)= \begin{cases}1 & \text { if } u \leq v \\
\frac{v}{u} & \text { otherwise }\end{cases}$ \\
\hline$T_{L K}(u, v)=\max (u+v-1,0)$ & $I_{L K}(u, v)=\min (1,1-u+v)$ \\
\hline$T_{D}(u, v)= \begin{cases}0 & \text { if }(u, v) \in\left[0,1\left[^{2}\right.\right. \\
\min (u, v) & \text { otherwise }\end{cases}$ & $I_{W B}(u, v)= \begin{cases}1 & \text { if }(u, v) \in\left[0,1\left[^{2}\right.\right. \\
v & \text { otherwise }\end{cases}$ \\
\hline$T_{n M}(u, v)= \begin{cases}0 & \text { if } u+v \leq 1 \\
\min (u, v) & \text { otherwise }\end{cases}$ & $I_{F D}(u, v)= \begin{cases}1 & \text { if } u \leq v \\
\max (1-u, v) & \text { otherwise }\end{cases}$ \\
\hline
\end{tabular}

Example 2. ([27]) Some basic $t$-conorms and their residual co-implications on $([0,1] ; \leq)$ (Table 2$)$ are defined by, for all $u, v \in[0,1]$,

Table 2. Some basic t-conorms and their residual co-implications on $([0,1] ; \leq)$.

\begin{tabular}{|c|c|}
\hline t-Conorms & Residual Co-Implications \\
\hline$S_{M}(u, v)=\max (u, v)$ & $J_{G D}(u, v)= \begin{cases}0 & \text { if } u \geq v \\
v & \text { otherwise }\end{cases}$ \\
\hline$S_{P}(u, v)=u+v-u \cdot v$ & $J_{G G}(u, v)= \begin{cases}0 & \text { if } u \geq v \\
\frac{v-u}{1-u} & \text { otherwise }\end{cases}$ \\
\hline$S_{L K}(u, v)=\min (u+v, 1)$ & $J_{L K}(u, v)=\max (0, v-u)$ \\
\hline$S_{D}(u, v)= \begin{cases}1 & \text { if }(u, v) \in] 0,1]^{2} \\
\max (u, v) & \text { otherwise }\end{cases}$ & $J_{W B}(u, v)= \begin{cases}0 & \text { if }(u, v) \in] 0,1]^{2} \\
v & \text { otherwise }\end{cases}$ \\
\hline$S_{n M}(u, v)= \begin{cases}1 & \text { if } u+v \geq 1 \\
\max (u, v) & \text { otherwise }\end{cases}$ & $J_{F D}(u, v)= \begin{cases}0 & \text { if } u \geq v \\
\min (1-u, v) & \text { otherwise }\end{cases}$ \\
\hline
\end{tabular}

3. The Lattice Structure of $\left(D^{*} ; \leq_{1}\right)$

Now we consider the set $D^{*}$ defined by,

$$
D^{*}=\left\{u=\left(u_{1}, u_{2}, u_{3}\right) \mid u_{1}, u_{2}, u_{3} \in[0,1]\right\} .
$$

As defined above, if $u \in D^{*}$, then $u$ has three components: The first component $u_{1}$, the second component $u_{2}$ and the third component $u_{3}$.

The order relation $\leq_{1}$ on $D^{*}$ can also be defined by, for all $u, v \in D^{*}$,

$$
u \leq_{1} v \text { if and only if } u_{1} \leq v_{1}, u_{2} \geq v_{2}, u_{3} \geq v_{3} .
$$

Proposition 1. $\left(D^{*} ; \leq_{1}\right)$ is a partially ordered set.

\section{Proof.}

(1) Reflexivity: $u \leq_{1} u$, for all $u \in D^{*}$.

(2) Anti-symmetry: If $u \leq_{1} v$ and $v \leq_{1} u$, then it is obvious that $u=v$, for all $u, v \in D^{*}$.

(3) Transitivity: If $u \leq_{1} v$ and $v \leq_{1} w$, then $u_{1} \leq w_{1}, u_{2} \geq w_{2}, u_{3} \geq w_{3}$, that is, $u \leq_{1} w$, for all $u, v, w \in D^{*}$. 
Proposition 2. The operations $\wedge_{1}$ and $\vee_{1}$ are defined by, for all $u, v \in D^{*}$,

$$
\begin{aligned}
& u \wedge_{1} v= \begin{cases}u & \text { if } u \leq_{1} v, \\
v & \text { if } v \leq_{1} u, \\
\left(\min \left(u_{1}, v_{1}\right), \max \left(u_{2}, v_{2}\right), \max \left(u_{3}, v_{3}\right)\right) & \text { otherwise. }\end{cases} \\
& u \vee_{1} v= \begin{cases}u & \text { if } v \leq_{1} u, \\
v & \text { if } u \leq_{1} v, \\
\left(\max \left(u_{1}, v_{1}\right), \min \left(u_{2}, v_{2}\right), \min \left(u_{3}, v_{3}\right)\right) & \text { otherwise. }\end{cases}
\end{aligned}
$$

Then $u \wedge_{1} v$ is called the greatest lower bound of $u, v$, denoted by $\inf (u, v) ; u \vee_{1} v$ is called the least upper bound of $u, v$, denoted by $\sup (u, v)$. That is, $\left(D^{*} ; \leq_{1}\right)$ is a lattice.

Proof. According to the definitions above, if either $u \leq_{1} v$ or $v \leq_{1} u, u \wedge_{1} v$ is the greatest lower bound of $u$ and $v$. Thus, $u \wedge_{1} v=\inf (u, v)$. Similarly, $u \vee_{1} v=\sup (u, v)$ can be obtained.

Now, we assume that neither $u \leq_{1} v$ nor $v \leq_{1} u$. According to the definitions above, $u \wedge_{1} v=$ $\left(\min \left(u_{1}, v_{1}\right), \max \left(u_{2}, v_{2}\right), \max \left(u_{3}, v_{3}\right)\right), u \vee_{1} v=\left(\max \left(u_{1}, v_{1}\right), \min \left(u_{2}, v_{2}\right), \min \left(u_{3}, v_{3}\right)\right)$.

(i) To prove $u \wedge_{1} v=\inf (u, v)$, we denote

$$
\kappa=\left(\kappa_{1}, \kappa_{2}, \kappa_{3}\right)=\left(\min \left(u_{1}, v_{1}\right), \max \left(u_{2}, v_{2}\right), \max \left(u_{3}, v_{3}\right)\right) .
$$

Since $u_{1} \geq \min \left(u_{1}, v_{1}\right)=\kappa_{1}, u_{2} \leq \max \left(u_{2}, v_{2}\right)=\kappa_{2}, u_{3} \leq \max \left(u_{3}, v_{3}\right)=\kappa_{3}, \kappa \leq_{1} u$. Similarly, we have $\kappa \leq_{1} v$. Thus, $\kappa$ is the lower bound of $u$ and $v$. Furthermore, $\kappa$ is the greatest lower bound of $u$ and $v$. In fact, assume $a=\left(a_{1}, a_{2}, a_{3}\right) \in D^{*}$ with the condition $a \leq_{1} u$ and $a \leq_{1} v$. Then, $a_{1} \leq u_{1}, a_{2} \geq$ $u_{2}, a_{3} \geq u_{3}$ and $a_{1} \leq v_{1}, a_{2} \geq v_{2}, a_{3} \geq v_{3}$. Therefore, $a_{1} \leq \min \left(u_{1}, v_{1}\right)=\kappa_{1}, a_{2} \geq \max \left(u_{2}, v_{2}\right)=\kappa_{2}$, $a_{3} \geq \max \left(u_{3}, v_{3}\right)=\kappa_{3}$. Hence, $a \leq_{1} \kappa$. To sum up, $\kappa=\left(\min \left(u_{1}, v_{1}\right), \max \left(u_{2}, v_{2}\right), \max \left(u_{3}, v_{3}\right)\right)$ is the greatest lower bound of $u$ and $v$.

(ii) To prove $u \vee_{1} v=\sup (u, v)$, we denote

$$
\omega=\left(\omega_{1}, \omega_{2}, \omega_{3}\right)=\left(\max \left(u_{1}, v_{1}\right), \min \left(u_{2}, v_{2}\right), \min \left(u_{3}, v_{3}\right)\right) .
$$

Since $u_{1} \leq \max \left(u_{1}, v_{1}\right)=\omega_{1}, u_{2} \geq \min \left(u_{2}, v_{2}\right)=\omega_{2}, u_{3} \geq \min \left(u_{3}, v_{3}\right)=\omega_{3}, u \leq_{1} \omega$. Similarly, we have $v \leq_{1} \omega$. Thus, $\omega$ is the upper bound of $u$ and $v$. Furthermore, $\omega$ is the least upper bound of $u$ and $v$. In fact, assume $b=\left(b_{1}, b_{2}, b_{3}\right) \in D^{*}$ with the condition $b \geq_{1} u$ and $b \geq_{1} v$. Then, $b_{1} \geq u_{1}, b_{2} \leq$ $u_{2}, b_{3} \leq u_{3}$ and $b_{1} \geq v_{1}, b_{2} \leq v_{2}, b_{3} \leq v_{3}$. Therefore, $b_{1} \geq \max \left(u_{1}, v_{1}\right)=\omega_{1}, b_{2} \leq \min \left(u_{2}, v_{2}\right)=\omega_{2}$, $b_{3} \leq \min \left(u_{3}, v_{3}\right)=\omega_{3}$. Hence, $b \geq_{1} \omega$. To sum up, $\omega=\left(\max \left(u_{1}, v_{1}\right), \min \left(u_{2}, v_{2}\right), \min \left(u_{3}, v_{3}\right)\right)$ is the least upper bound of $u$ and $v$.

(i) and (ii) show that $u \wedge_{1} v=\inf (u, v), u \vee_{1} v=\sup (u, v)$, for all $u, v \in D^{*}$. Then $\left(D^{*} ; \leq_{1}\right)$ is a lattice.

The first, second and third projection mapping $p r_{1}, p r_{2}$ and $p r_{3}$ on $D^{*}$ are defined as follows, $p r_{1}(u)=u_{1}, p r_{2}(u)=u_{2}$ and $p r_{3}(u)=u_{3}$, for all $u \in D^{*}$.

Proposition $3 .\left(D^{*} ; \leq_{1}\right)$ is a complete lattice.

Proof. Let $B$ be a nonempty subset of $D^{*}$, we have

$$
\inf B=\left(\inf p r_{1} B, \inf p r_{2} B, \inf p r_{3} B\right),
$$

where $\inf \operatorname{pr}_{1} B=\inf \left\{u_{1} \mid u_{1} \in[0,1], \exists u=\left(u_{1}, u_{2}, u_{3}\right) \in B\right\}, \inf p r_{2} B=\sup \left\{u_{2} \mid u_{2} \in[0,1], \exists u=\right.$ $\left.\left(u_{1}, u_{2}, u_{3}\right) \in B\right\}, \inf \operatorname{pr}_{3} B=\sup \left\{u_{3} \mid u_{3} \in[0,1], \exists u=\left(u_{1}, u_{2}, u_{3}\right) \in B\right\}$.

And 
$\sup B=\left(\sup p r_{1} B, \sup p r_{2} B, \sup p r_{3} B\right)$,

where $\sup p r_{1} B=\sup \left\{u_{1} \mid u_{1} \in[0,1], \exists u=\left(u_{1}, u_{2}, u_{3}\right) \in B\right\}, \sup \operatorname{pr}_{2} B=\inf \left\{u_{2} \mid u_{2} \in[0,1], \exists u=\right.$ $\left.\left(u_{1}, u_{2}, u_{3}\right) \in B\right\}, \sup \operatorname{pr}_{3} B=\inf \left\{u_{3} \mid u_{3} \in[0,1], \exists u=\left(u_{1}, u_{2}, u_{3}\right) \in B\right\}$.

The maximum and minimum of $D^{*}$ are denoted by $1_{D^{*}}=(1,0,0)$ and $0_{D^{*}}=(0,1,1)$, respectively.

Note that, if $u$ and $v$ are incomparable with respect to $\leq_{1}$, for all $u, v \in D^{*}$, then the relationship between $u$ and $v$ can be denoted as $u \|_{\leq_{1}} v$.

Obviously, each neutrosophic set $M=\left\{\left\langle u, T_{M}(u), I_{M}(u), F_{M}(u)\right\rangle \mid u \in U\right\}$ corresponds to a $D^{*}$-fuzzy set. That is, there exits a mapping

$$
M: U \rightarrow D^{*} ; u \longmapsto\left(T_{M}(u), I_{M}(u), F_{M}(u)\right) .
$$

Based on the relationship between neutrosophic sets and $D^{*}$-fuzzy sets, the triple formed by the three membership degrees of neutrosophic sets is an element of $D^{*}$. Therefore, we can obtain more compact formulas for neutrosophic sets, analyze and extend some operators defined in the fuzzy case for neutrosophic sets by using the lattice $\left(D^{*} ; \leq_{1}\right)$.

For example, the intersection of two neutrosophic sets $M$ and $N$ in a universe $U$ is defined as

$$
M \cap_{1} N=\left\{\left\langle u, \min \left(T_{M}(u), T_{N}(u)\right), \max \left(I_{M}(u), I_{N}(u)\right), \max \left(F_{M}(u), F_{N}(u)\right)\right\rangle \mid u \in U\right\} .
$$

Using the lattice $\left(D^{*} ; \leq_{1}\right)$, we can get, for all $u \in U$,

$\left(M \cap_{1} N\right)(u)=\left(\min \left(T_{M}(u), T_{N}(u)\right), \max \left(I_{M}(u), I_{N}(u)\right), \max \left(F_{M}(u), F_{N}(u)\right)\right)=M(u) \wedge_{1} N(u)$.

Definition 6. The complement of $u$ is defined by, for all $u \in D^{*}$,

$$
u^{c}=\left(u_{3}, 1-u_{2}, u_{1}\right) .
$$

Proposition 4. Let $u, v, w \in D^{*}$. Then

(1) $u \wedge_{1} u=u, u \vee_{1} u=u$;

(2) $u \wedge_{1} v=v \wedge_{1} u, u \vee_{1} v=v \vee_{1} u$;

(3) $\left(u \wedge_{1} v\right) \wedge_{1} w=u \wedge_{1}\left(v \wedge_{1} w\right),\left(u \vee_{1} v\right) \vee_{1} w=u \vee_{1}\left(v \vee_{1} w\right)$

(4) $u \wedge_{1}\left(v \vee_{1} u\right)=u, u \vee_{1}\left(v \wedge_{1} u\right)=u$;

(5) $u \leq_{1} v$ if and only if $u \vee_{1} v=v, u \wedge_{1} v=u$;

(6) $\left(u^{c}\right)^{c}=u$.

Proposition 5. Let $u, v \in D^{*}$. Then

(1) $\left(u \wedge_{1} v\right)^{c}=u^{c} \vee_{1} v^{c}$;

(2) $\left(u \vee_{1} v\right)^{c}=u^{c} \wedge_{1} v^{c}$.

Proof. (1) Suppose $u, v \in D^{*}$. If $u \leq_{1} v$, that is, $u_{1} \leq v_{1}, u_{2} \geq v_{2}, u_{3} \geq v_{3}$. By Definition 6, we have $u^{c} \geq_{1} v^{c}$. Thus, $\left(u \wedge_{1} v\right)^{c}=u^{c} \vee_{1} v^{c}$. Similarly, if $u \geq_{1} v$, then $u^{c} \leq_{1} v^{c}$ and $\left(u \wedge_{1} v\right)^{c}=u^{c} \vee_{1} v^{c}$. If $u \|_{\leq_{1}} v$, then $u \wedge_{1} v=\left(\min \left(u_{1}, v_{1}\right), \max \left(u_{2}, v_{2}\right), \max \left(u_{3}, v_{3}\right)\right)$. Thus, $\left(u \wedge_{1} v\right)^{c}=\left(\max \left(u_{3}, v_{3}\right)\right.$, $\left.1-\max \left(u_{2}, v_{2}\right), \min \left(u_{1}, v_{1}\right)\right)$. Since $u^{c} \vee_{1} v^{c}=\left(u_{3}, 1-u_{2}, u_{1}\right) \vee_{1}\left(v_{3}, 1-v_{2}, v_{1}\right)=\left(\max \left(u_{3}, v_{3}\right)\right.$, $\left.\min \left(1-u_{2}, 1-v_{2}\right), \min \left(u_{1}, v_{1}\right)\right)=\left(\max \left(u_{3}, v_{3}\right), 1-\max \left(u_{2}, v_{2}\right), \min \left(u_{1}, v_{1}\right)\right)$. Hence, $\left(u \wedge_{1} v\right)^{c}=$ $u^{c} \vee_{1} v^{c}$.

(2) Similarly, we can get $\left(u \vee_{1} v\right)^{c}=u^{c} \wedge_{1} v^{c}$.

Proposition 6. The system $\left(D^{*} ; \wedge_{1}, \vee_{1},{ }^{c}, 0_{D^{*}}, 1_{D^{*}}\right)$ is a De Morgan algebra.

Proof. By Propositions $1-5$ and the definition of the generalized De Morgan algebra [14,55], we can get that $\left(D^{*} ; \wedge_{1}, \vee_{1},{ }^{c}, 0_{D^{*}}, 1_{D^{*}}\right)$ is a generalized De Morgan algebra. Furthermore, we can prove that $\left(D^{*} ; \wedge_{1}, \vee_{1},{ }^{c}, 0_{D^{*}}, 1_{D^{*}}\right)$ is a distributive lattice, that is, for all $u, v, w \in D^{*}$ such that $u \wedge_{1}\left(v \vee_{1} w\right)=$ $\left(u \wedge_{1} v\right) \vee_{1}\left(u \wedge_{1} w\right)$. 
(1) For all $u, v, w \in D^{*}$, if any two of them are comparable, then there are six situations as follows:

Case 1: If $u \leq_{1} v \leq_{1} w$, then $u \wedge_{1}\left(v \vee_{1} w\right)=u \wedge_{1} w=u,\left(u \wedge_{1} v\right) \vee_{1}\left(u \wedge_{1} w\right)=u \vee_{1} u=u$.

Case 2: If $u \leq_{1} w \leq_{1} v$, then $u \wedge_{1}\left(v \vee_{1} w\right)=u \wedge_{1} v=u,\left(u \wedge_{1} v\right) \vee_{1}\left(u \wedge_{1} w\right)=u \vee_{1} u=u$.

Case 3: If $w \leq_{1} u \leq_{1} v$, then $u \wedge_{1}\left(v \vee_{1} w\right)=u \wedge_{1} v=u,\left(u \wedge_{1} v\right) \vee_{1}\left(u \wedge_{1} w\right)=u \vee_{1} w=u$.

Case 4: If $v \leq_{1} u \leq_{1} w$, then $u \wedge_{1}\left(v \vee_{1} w\right)=u \wedge_{1} w=u,\left(u \wedge_{1} v\right) \vee_{1}\left(u \wedge_{1} w\right)=v \vee_{1} u=u$.

Case 5: If $w \leq_{1} v \leq_{1} u$, then $u \wedge_{1}\left(v \vee_{1} w\right)=u \wedge_{1} v=v,\left(u \wedge_{1} v\right) \vee_{1}\left(u \wedge_{1} w\right)=v \vee_{1} w=v$.

Case 6: If $v \leq_{1} w \leq_{1} u$, then $u \wedge_{1}\left(v \vee_{1} w\right)=u \wedge_{1} w=w,\left(u \wedge_{1} v\right) \vee_{1}\left(u \wedge_{1} w\right)=v \vee_{1} w=w$.

Thus, $u \wedge_{1}\left(v \vee_{1} w\right)=\left(u \wedge_{1} v\right) \vee_{1}\left(u \wedge_{1} w\right)$.

(2) For all $u, v, w \in D^{*}$, if at least two of them are not comparable, then $u \wedge_{1}\left(v \vee_{1} w\right)=u \wedge_{1}$ $\left(\max \left(v_{1}, w_{1}\right), \min \left(v_{2}, w_{2}\right), \min \left(v_{3}, w_{3}\right)\right)=\left(\min \left(u_{1}, \max \left(v_{1}, w_{1}\right)\right), \max \left(u_{2}, \min \left(v_{2}, w_{2}\right)\right), \max \left(u_{3}\right.\right.$, $\left.\left.\min \left(v_{3}, w_{3}\right)\right)\right)=\left(\max \left(\min \left(u_{1}, v_{1}\right), \min \left(u_{1}, w_{1}\right)\right), \min \left(\max \left(u_{2}, v_{2}\right), \max \left(u_{2}, w_{2}\right)\right), \min \left(\max \left(u_{3}, v_{3}\right)\right.\right.$, $\left.\left.\max \left(u_{3}, w_{3}\right)\right)\right)=\left(\min \left(u_{1}, v_{1}\right), \max \left(u_{2}, v_{2}\right), \max \left(u_{3}, v_{3}\right)\right) \vee\left(\min \left(u_{1}, w_{1}\right), \max \left(u_{2}, w_{2}\right), \max \left(u_{3}, w_{3}\right)\right)$ $=\left(u \wedge_{1} v\right) \vee_{1}\left(u \wedge_{1} w\right)$.

Therefore, $\left(D^{*} ; \wedge_{1}, \vee_{1}{ }^{c}, 0_{D^{*}}, 1_{D^{*}}\right)$ is a De Morgan algebra.

Considering the second type inclusion relation on neutrosophic sets which is dual of the first type inclusion relation, we get that $\left(D^{*} ; \wedge_{2}, \vee_{2}{ }^{c},(0,0,1),(1,1,0)\right)$ is also a De Morgan algebra.

From this, Proposition 2.2 (see [14]) can be easily proved by using Proposition 6. That is, Proposition 2.2 (see [14]) is a corollary of Proposition 6.

In short, in combination with the conclusions given in [14], we find that neutrosophic net is different from intuitionistic fuzzy set.

\section{Neutrosophic t-Norms and De Morgan Neutrosophic Triples}

Section 3 proposes that $\left(D^{*} ; \leq_{1}\right)$ is a complete lattice, Section 4 will introduce the notions of neutrosophic t-norms (t-conorms) on $\left(D^{*} ; \leq_{1}\right)$.

Definition 7. A neutrosophic t-norm is a function $\mathscr{T}:\left(D^{*}\right)^{2} \rightarrow D^{*}$ that satisfies the following conditions, for all $u, v, w \in D^{*}$ :

(NT1) $\mathscr{T}(u, v)=\mathscr{T}(v, u)$;

(NT2) $\mathscr{T}(u, \mathscr{T}(v, w))=\mathscr{T}(v, \mathscr{T}(u, w))$;

(NT3) $\mathscr{T}(u, v) \leq_{1} \mathscr{T}\left(u^{\prime}, v^{\prime}\right)$, where $u \leq_{1} u^{\prime}, v \leq_{1} v^{\prime}$;

(NT4) $\mathscr{T}\left(u, 1_{D^{*}}\right)=u$.

Definition 8. A neutrosophic t-conorm is a function $\mathscr{S}:\left(D^{*}\right)^{2} \rightarrow D^{*}$ that satisfies the following conditions, for all $u, v, w \in D^{*}$ :

(NS1) $\mathscr{S}(u, v)=\mathscr{S}(v, u)$;

(NS2) $\mathscr{S}(u, \mathscr{S}(v, w))=\mathscr{S}(v, \mathscr{S}(u, w))$;

(NS3) $\mathscr{S}(u, v) \leq_{1} \mathscr{S}\left(u^{\prime}, v^{\prime}\right)$, where $u \leq_{1} u^{\prime}, v \leq_{1} v^{\prime}$;

(NS4) $\mathscr{S}\left(u, 0_{D^{*}}\right)=u$.

Some basic neutrosophic t-norms (t-conorms) on $\left(D^{*} ; \leq_{1}\right)$ are presented as follows:

Example 3. Some neutrosophic t-norms are defined by, for all $u, v \in D^{*}$ :

(1) $\mathscr{T}_{M}(u, v)=\left(T_{M}\left(u_{1}, v_{1}\right), S_{M}\left(u_{2}, v_{2}\right), S_{M}\left(u_{3}, v_{3}\right)\right)$;

(2) $\mathscr{T}_{P}(u, v)=\left(T_{P}\left(u_{1}, v_{1}\right), S_{P}\left(u_{2}, v_{2}\right), S_{P}\left(u_{3}, v_{3}\right)\right)$;

(3) $\mathscr{T}_{L K}(u, v)=\left(T_{L K}\left(u_{1}, v_{1}\right), S_{L K}\left(u_{2}, v_{2}\right), S_{L K}\left(u_{3}, v_{3}\right)\right)$; 
(4) $\mathscr{T}_{D}(u, v)=\left(T_{D}\left(u_{1}, v_{1}\right), S_{D}\left(u_{2}, v_{2}\right), S_{D}\left(u_{3}, v_{3}\right)\right)$;

(5) $\mathscr{T}_{n M}(u, v)=\left(T_{n M}\left(u_{1}, v_{1}\right), S_{n M}\left(u_{2}, v_{2}\right), S_{n M}\left(u_{3}, v_{3}\right)\right)$;

(6) $\mathscr{T}(u, v)=\left(T_{P}\left(u_{1}, v_{1}\right), S_{L K}\left(u_{2}, v_{2}\right), S_{D}\left(u_{3}, v_{3}\right)\right)$;

(7) $\mathscr{T}(u, v)=\left(T_{D}\left(u_{1}, v_{1}\right), S_{P}\left(u_{2}, v_{2}\right), S_{P}\left(u_{3}, v_{3}\right)\right)$;

(8) $\mathscr{T}(u, v)=\left(T_{L K}\left(u_{1}, v_{1}\right), S_{L K}\left(u_{2}, v_{2}\right), S_{M}\left(u_{3}, v_{3}\right)\right)$;

(9) $\mathscr{T}(u, v)=\left(T_{M}\left(u_{1}, v_{1}\right), S_{P}\left(u_{2}, v_{2}\right), S_{M}\left(u_{3}, v_{3}\right)\right)$.

Example 4. Some neutrosophic $t$-conorms are defined by, for all $u, v \in D^{*}$ :

(1) $\mathscr{S}_{M}(u, v)=\left(S_{M}\left(u_{1}, v_{1}\right), T_{M}\left(u_{2}, v_{2}\right), T_{M}\left(u_{3}, v_{3}\right)\right)$;

(2) $\mathscr{S}_{P}(u, v)=\left(S_{P}\left(u_{1}, v_{1}\right), T_{P}\left(u_{2}, v_{2}\right), T_{P}\left(u_{3}, v_{3}\right)\right)$;

(3) $\mathscr{S}_{L K}(u, v)=\left(S_{L K}\left(u_{1}, v_{1}\right), T_{L K}\left(u_{2}, v_{2}\right), T_{L K}\left(u_{3}, v_{3}\right)\right)$;

(4) $\mathscr{S}_{D}(u, v)=\left(S_{D}\left(u_{1}, v_{1}\right), T_{D}\left(u_{2}, v_{2}\right), T_{D}\left(u_{3}, v_{3}\right)\right)$;

(5) $\mathscr{S}_{n M}(u, v)=\left(S_{n M}\left(u_{1}, v_{1}\right), T_{n M}\left(u_{2}, v_{2}\right), T_{n M}\left(u_{3}, v_{3}\right)\right)$;

(6) $\mathscr{S}(u, v)=\left(S_{M}\left(u_{1}, v_{1}\right), T_{P}\left(u_{2}, v_{2}\right), T_{L K}\left(u_{3}, v_{3}\right)\right)$;

(7) $\mathscr{S}(u, v)=\left(S_{P}\left(u_{1}, v_{1}\right), T_{L K}\left(u_{2}, v_{2}\right), T_{L K}\left(u_{3}, v_{3}\right)\right)$;

(8) $\mathscr{S}(u, v)=\left(S_{L K}\left(u_{1}, v_{1}\right), T_{L K}\left(u_{2}, v_{2}\right), T_{M}\left(u_{3}, v_{3}\right)\right)$;

(9) $\mathscr{S}(u, v)=\left(S_{D}\left(u_{1}, v_{1}\right), T_{P}\left(u_{2}, v_{2}\right), T_{n M}\left(u_{3}, v_{3}\right)\right)$.

Furthermore, the representation theorems of neutrosophic t-norms (t-conorms) are proposed as follows:

Theorem 1. Let $\mathscr{T}$ be a binary operation on $D^{*}$. Then, for all $u, v \in D^{*}$,

$$
\mathscr{T}(u, v)=\left(T\left(u_{1}, v_{1}\right), S_{1}\left(u_{2}, v_{2}\right), S_{2}\left(u_{3}, v_{3}\right)\right)
$$

is a neutrosophic t-norm, where $S_{1}, S_{2}$ are t-conorms, $T$ is a $t$-norm on $[0,1]$.

Proof. (NT1) Let $S_{1}, S_{2}$ be two t-conorms, $T$ is a t-norm on $[0,1]$. Since $T\left(u_{1}, v_{1}\right)=T\left(v_{1}, u_{1}\right)$, $S_{1}\left(u_{2}, v_{2}\right)=S_{1}\left(v_{2}, u_{2}\right), S_{2}\left(u_{3}, v_{3}\right)=S_{2}\left(v_{3}, u_{3}\right), \mathscr{T}(u, v)=\mathscr{T}(v, u)$, for all $u, v \in D^{*}$.

(NT2) $\mathscr{T}\left(1_{D^{*}}, u\right)=\left(T\left(1, u_{1}\right), S_{1}\left(0, u_{2}\right), S_{2}\left(0, u_{3}\right)\right)=\left(u_{1}, u_{2}, u_{3}\right)=u$, for all $u \in D^{*}$.

(NT3) For all $u, u^{\prime}, v, v^{\prime} \in D^{*}$ with the condition $u \leq_{1} u^{\prime}, v \leq_{1} v^{\prime}$, we have $T\left(u_{1}, v_{1}\right) \leq T\left(u_{1}^{\prime}, v_{1}^{\prime}\right)$, $S_{1}\left(u_{2}, v_{2}\right) \geq S_{1}\left(u_{2}^{\prime}, v_{2}^{\prime}\right), S_{2}\left(u_{3}, v_{3}\right) \geq S_{2}\left(u_{3}^{\prime}, v_{3}^{\prime}\right)$. Therefore, $\mathscr{T}(u, v) \leq_{1} \mathscr{T}\left(u^{\prime}, v^{\prime}\right)$.

(NT4) $\mathscr{T}(u, \mathscr{T}(v, w))=\mathscr{T}\left(u,\left(T\left(v_{1}, w_{1}\right), s_{1}\left(v_{2}, w_{2}\right), S_{2}\left(v_{3}, w_{3}\right)\right)\right)=\left(T\left(u_{1}, T\left(v_{1}, w_{1}\right)\right), S_{1}\left(u_{2}\right.\right.$, $\left.\left.S_{1}\left(v_{2}, w_{2}\right)\right), S_{2}\left(u_{3}, S_{2}\left(v_{3}, w_{3}\right)\right)\right)=\left(T\left(v_{1}, T\left(u_{1}, w_{1}\right)\right), S_{1}\left(v_{2}, S_{1}\left(u_{2}, w_{2}\right)\right), S_{2}\left(v_{3}, S_{2}\left(u_{3}, w_{3}\right)\right)\right)=\mathscr{T}(v$, $\mathscr{T}(u, w))$, for all $u, v, w \in D^{*}$.

Hence, $\mathscr{T}(u, v)$ is a neutrosophic t-norm.

Theorem 2. Let $\mathscr{S}:\left(D^{*}\right)^{2} \rightarrow D^{*}$ be a mapping. Then, for all $u, v \in D^{*}$,

$$
\mathscr{S}(u, v)=\left(S\left(u_{1}, v_{1}\right), T_{1}\left(u_{2}, v_{2}\right), T_{2}\left(u_{3}, v_{3}\right)\right)
$$

is a neutrosophic t-conorm, where $S$ is a t-conorm, $T_{1}, T_{2}$ are $t$-norms on $[0,1]$.

Proof. The proof is similar to that of Theorem 1.

Theorem 1 proposes a way to construct neutrosophic t-norms on $D^{*}$ with $\mathrm{t}$-norms and $\mathrm{t}$-conorms which are defined on $[0,1]$. Unfortunately, the converse is not always true. It is not always possible to find two t-conorms $S_{1}, S_{2}$, a t-norm $T$ on $[0,1]$ such that $\mathscr{T}=\left(T, S_{1}, S_{2}\right)$.

To distinguish these two kinds of neutrosophic t-norms, we introduce the notions of representable neutrosophic t-norms.

Definition 9. A neutrosophic $t$-norm $\mathscr{T}$ is called representable, if and only if, there exist two t-conorms $S_{1}, S_{2}$ and a t-norm $T$ on $[0,1]$ satisfying, for any $u, v \in D^{*}$, 


$$
\mathscr{T}(u, v)=\left(T\left(u_{1}, v_{1}\right), S_{1}\left(u_{2}, v_{2}\right), S_{2}\left(u_{3}, v_{3}\right)\right)
$$

Definition 10. A neutrosophic t-norm $\mathscr{T}$ is called standard representable, if and only if, there exists a $t$-norm $T$ and a t-conorm $S$ on $[0,1]$ satisfying, for any $u, v \in D^{*}$,

$$
\mathscr{T}(u, v)=\left(T\left(u_{1}, v_{1}\right), S\left(u_{2}, v_{2}\right), S\left(u_{3}, v_{3}\right)\right) .
$$

Definition 11. A N-dual representable neutrosophic t-norm $\mathscr{T}$ defined by, for any $u, v \in D^{*}$,

$$
\mathscr{T}(u, v)=\left(T\left(u_{1}, v_{1}\right), S\left(u_{2}, v_{2}\right), S\left(u_{3}, v_{3}\right)\right) .
$$

where $T$ is a t-norm on $[0,1]$ and $S$ is the $N$-dual t-conorm of $T$, that is, $T(u, v)=1-S(1-u, 1-v)$.

Definition 12. A first $N$-dual representable neutrosophic t-norm $\mathscr{T}$ defined by, for any $u, v \in D^{*}$,

$$
\mathscr{T}(u, v)=\left(T\left(u_{1}, v_{1}\right), S_{1}\left(u_{2}, v_{2}\right), S_{2}\left(u_{3}, v_{3}\right)\right) .
$$

where $T$ is a t-norm on $[0,1]$ and $S_{1}$ is the $N$-dual t-conorm of $T, S_{2}$ is a t-conorm on $[0,1]$.

Definition 13. A second $N$-dual representable neutrosophic $t$-norm $\mathscr{T}$ defined by, for any $u, v \in D^{*}$,

$$
\mathscr{T}(u, v)=\left(T\left(u_{1}, v_{1}\right), S_{1}\left(u_{2}, v_{2}\right), S_{2}\left(u_{3}, v_{3}\right)\right)
$$

where $T$ is a $t$-norm on $[0,1]$ and $S_{2}$ is the $N$-dual $t$-conorm of $T, S_{1}$ is a t-conorm on $[0,1]$.

Notice that the $\mathrm{N}$-dual representable neutrosophic t-norms are not only the standard representable neutrosophic t-norms, but also the first $\mathrm{N}$-dual representable neutrosophic t-norms and the second $\mathrm{N}$-dual representable neutrosophic t-norms. Those neutrosophic t-norms presented in Example 3 are all representable neutrosophic t-norms, and (1)-(5) are N-dual representable neutrosophic t-norms, (8) is a first $\mathrm{N}$-dual representable neutrosophic $\mathrm{t}$-norm, (9) is a second $\mathrm{N}$-dual representable neutrosophic t-norm.

Definition 14. A neutrosophic t-conorm $\mathscr{S}$ is called representable, if and only if, there exists a t-conorm $S$ and two t-norms $T_{1}, T_{2}$ on $[0,1]$ satisfying, for any $u, v \in D^{*}$,

$$
\mathscr{S}(u, v)=\left(S\left(u_{1}, v_{1}\right), T_{1}\left(u_{2}, v_{2}\right), T_{2}\left(u_{3}, v_{3}\right)\right) .
$$

For neutrosophic $t$-conorms, the rest of the related concepts can be obtained by contrasting with Definitions 10-13 of neutrosophic t-norms above.

The following propositions present a method for constructing new representable neutrosophic $\mathrm{t}$-norms (t-conorms) with intuitionistic fuzzy t-norms (t-conorms).

Proposition 7. Let $T(x, y)$ be a representable intuitionistic fuzzy t-norm: $T(x, y)=\left(t\left(x_{1}, y_{1}\right), s_{2}\left(x_{3}, y_{3}\right)\right)$, for all $x=\left(x_{1}, x_{3}\right), y=\left(y_{1}, y_{3}\right) \in L^{*}$, where $t$ is a $t$-norm, $s_{2}$ is a $t$-conorm on $[0,1]$. Assume that $s_{1}$ is a t-conorm on $[0,1]$, satisfying, $0 \leq t\left(u_{1}, v_{1}\right)+s_{1}\left(u_{2}, v_{2}\right)+s_{2}\left(u_{3}, v_{3}\right) \leq 3$. Then $\mathscr{T}(u, v)=$ $\left(t\left(u_{1}, v_{1}\right), s_{1}\left(u_{2}, v_{2}\right), s_{2}\left(u_{3}, v_{3}\right)\right)$ is a representable neutrosophic $t$-norm, for any $u, v \in D^{*}$.

Proposition 8. Let $S(x, y)$ be a representable intuitionistic fuzzy t-conorm: $S(x, y)=\left(s\left(x_{1}, y_{1}\right), t_{2}\left(x_{3}, y_{3}\right)\right)$, for all $u=\left(x_{1}, x_{3}\right), v=\left(y_{1}, y_{3}\right) \in L^{*}$, where $s$ is a $t$-conorm, $t_{2}$ is a $t$-norm on $[0,1]$. Suppose that $t_{1}$ is a t-norm on $[0,1]$, satisfying, $0 \leq s\left(u_{1}, v_{1}\right)+t_{1}\left(u_{2}, v_{2}\right)+t_{2}\left(u_{3}, v_{3}\right) \leq 3$. Then $\mathscr{S}(u, v)=$ $\left(s\left(u_{1}, v_{1}\right), t_{1}\left(u_{2}, v_{2}\right), t_{2}\left(u_{3}, v_{3}\right)\right)$ is a representable neutrosophic $t$-conorm, for all $u, v \in D^{*}$.

De Morgan triple is the perfect combination of a fuzzy t-norm, a fuzzy t-conorm and a fuzzy negator because it describes the duality of a fuzzy t-norm and a fuzzy t-conorm with respect to a fuzzy negator. Thus, it is necessary to discuss De Morgan neutrosophic triples. First of all, neutrosophic negators as the extension of fuzzy negators, as well as intuitionistic negators can be defined as follows: 
Definition 15. A neutrosophic negator is a function $\mathscr{N}: D^{*} \rightarrow D^{*}$ that satisfies the following conditions:

(NN1) $\mathscr{N}(u) \geq_{1} \mathscr{N}(v)$, for all $u, v \in D^{*}$ such that $u \leq_{1} v$;

(NN2) $\mathscr{N}\left(0_{D^{*}}\right)=1_{D^{*}}$;

(NN3) $\mathscr{N}\left(1_{D^{*}}\right)=0_{D^{*}}$.

If $\mathscr{N}(\mathscr{N}(u))=u$, for all $u \in D^{*}$, then $\mathscr{N}$ is called an involutive neutrosophic negator.

The mapping $\mathscr{N}_{s}: D^{*} \rightarrow D^{*}$ defined by, for all $\left(u_{1}, u_{2}, u_{3}\right) \in D^{*}$,

$$
\mathscr{N}_{\mathcal{S}}\left(u_{1}, u_{2}, u_{3}\right)=\left(u_{3}, 1-u_{2}, u_{1}\right)
$$

is an involutive neutrosophic negator. Then we call it the standard neutrosophic negator. Of course, $\mathscr{N}(u)=\left(u_{3}, 1-u_{3}, u_{1}\right), \mathscr{N}(u)=\left(u_{3}, u_{1}, u_{1}\right)$ are neutrosophic negators.

Definition 16. Let $\mathscr{T}$ be a neutrosophic t-norm, $\mathscr{S}$ be a neutrosophic $t$-conorm, $\mathscr{N}$ be a neutrosophic negator. The triple $(\mathscr{T}, \mathscr{N}, \mathscr{S})$ satisfied the following conditions, for all $u, v \in D^{*}$,

$$
\begin{aligned}
& \mathscr{N}(\mathscr{S}(u, v))=\mathscr{T}(\mathscr{N}(u), \mathscr{N}(v)) ; \\
& \mathscr{N}(\mathscr{T}(u, v))=\mathscr{S}(\mathscr{N}(u), \mathscr{N}(v))
\end{aligned}
$$

is called a De Morgan neutrosophic triple. Moreover, $\mathscr{T}$ and $\mathscr{S}$ are dual with respect to $\mathscr{N}$.

Theorem 3. Let $\mathscr{N}$ be an involutive neutrosophic negator.

(1) If $\mathscr{S}$ is a neutrosophic t-conorm, then the operator $\mathscr{T}$ defined by

$$
\mathscr{T}(u, v)=\mathscr{N}(\mathscr{S}(\mathscr{N}(u), \mathscr{N}(v))),
$$

is a neutrosophic t-norm. Furthermore, $(\mathscr{T}, \mathscr{N}, \mathscr{S})$ is a De Morgan neutrosophic triple.

(2) If $\mathscr{T}$ is a neutrosophic $t$-norm, then the operator $\mathscr{S}$ defined by

$$
\mathscr{S}(u, v)=\mathscr{N}(\mathscr{T}(\mathscr{N}(u), \mathscr{N}(v))),
$$

is a neutrosophic t-conorm. Furthermore, $(\mathscr{T}, \mathscr{N}, \mathscr{S})$ is a De Morgan neutrosophic triple.

Proof. (1) Let $\mathscr{N}$ be an involutive neutrosophic negator, $\mathscr{S}$ be a neutrosophic t-conorm.

(NT1) For any $u, v \in D^{*}, \mathscr{T}(u, v)=\mathscr{N}(\mathscr{S}(\mathscr{N}(u), \mathscr{N}(v)))=\mathscr{N}(\mathscr{S}(\mathscr{N}(v), \mathscr{N}(u)))=\mathscr{T}(v, u)$, because $\mathscr{S}$ is commutative. Thus, $\mathscr{T}$ is commutative.

(NT2) For any $u, v, w \in D^{*}, \mathscr{T}(u, \mathscr{T}(v, w))=\mathscr{T}(u, \mathscr{N}(\mathscr{S}(\mathscr{N}(v), \mathscr{N}(w))))=$ $\mathscr{N}(\mathscr{S}(\mathscr{N}(u), \mathscr{N}(\mathscr{N}(\mathscr{S}(\mathscr{N}(v), \mathscr{N}(w)))))=\mathscr{N}(\mathscr{S}(\mathscr{N}(u), \mathscr{S}(\mathscr{N}(v), \mathscr{N}(w))))=\mathscr{N}(\mathscr{S}(\mathscr{N}(v)$, $\mathscr{S}(\mathscr{N}(u), \mathscr{N}(w))))=\mathscr{T}(v, \mathscr{T}(u, w))$, because $\mathscr{S}$ is associative and $\mathscr{N}$ is involutive. Thus, $\mathscr{T}$ is associative.

(NT3) Let $u, u^{\prime}, v, v^{\prime} \in D^{*}$ with the condition $u \leq_{1} u^{\prime}, v \leq_{1} v^{\prime}$. Then $\mathscr{N}(u) \geq_{1} \mathscr{N}\left(u^{\prime}\right)$, $\mathscr{N}(v) \geq_{1} \mathscr{N}\left(v^{\prime}\right)$, because $\mathscr{N}$ is non-increasing. Since $\mathscr{S}$ is non-decreasing in its every variable, $\mathscr{S}(\mathscr{N}(u), \mathscr{N}(v)) \geq_{1} \mathscr{S}\left(\mathscr{N}\left(u^{\prime}\right), \mathscr{N}\left(v^{\prime}\right)\right)$. Thus, $\mathscr{N}(\mathscr{S}(\mathscr{N}(u), \mathscr{N}(v))) \leq_{1} \mathscr{N}\left(\mathscr{S}\left(\mathscr{N}\left(u^{\prime}\right), \mathscr{N}\left(v^{\prime}\right)\right)\right)$, that is, $\mathscr{T}(u, v) \leq_{1} \mathscr{T}\left(u^{\prime}, v^{\prime}\right)$. Hence, $\mathscr{T}$ is non-decreasing.

(NT4) For any $u \in D^{*}, \mathscr{T}\left(u, 1_{D^{*}}\right)=\mathscr{N}\left(\mathscr{S}\left(\mathscr{N}(u), 0_{D^{*}}\right)\right)=\mathscr{N}(\mathscr{N}(u))=u$.

Therefore, $\mathscr{T}$ is a neutrosophic t-norm.

Furthermore, $(\mathscr{T}, \mathscr{N}, \mathscr{S})$ is a De Morgan neutrosophic triple.

(2) Similarly, assume that $\mathscr{T}$ is a neutrosophic t-norm, $\mathscr{S}$ can be proved to be a neutrosophic $\mathrm{t}$-conorm and $(\mathscr{T}, \mathscr{N}, \mathscr{S})$ will be a De Morgan neutrosophic triple.

Proposition 9. Suppose that $(\mathscr{T}, \mathscr{N}, \mathscr{S})$ is a De Morgan neutrosophic triple, $\mathscr{N}$ is a standard neutrosophic negator. Then, for all $u \in D^{*}$, 
(1) $\mathscr{T}\left(u, 1_{D^{*}}\right)=u$ if and only if $\mathscr{S}\left(u, 0_{D^{*}}\right)=u$.

(2) $\mathscr{T}\left(u, 1_{D^{*}}\right)=\left(u_{1}, 0, u_{3}\right)$ if and only if $\mathscr{S}\left(u, 0_{D^{*}}\right)=\left(u_{1}, 1, u_{3}\right)$.

(3) $\mathscr{T}\left(u, 1_{D^{*}}\right)=\left(u_{1}, 1, u_{3}\right)$ if and only if $\mathscr{S}\left(u, 0_{D^{*}}\right)=\left(u_{1}, 0, u_{3}\right)$.

Example 5. Some neutrosophic t-norms and neutrosophic t-conorms are dual with respect to $\mathscr{N}_{s}$.

(1) $\mathscr{T}_{M}(u, v)=\left(T_{M}\left(u_{1}, v_{1}\right), S_{M}\left(u_{2}, v_{2}\right), S_{M}\left(u_{3}, v_{3}\right)\right), \mathscr{S}_{M}(u, v)=\left(S_{M}\left(u_{1}, v_{1}\right), T_{M}\left(u_{2}, v_{2}\right)\right.$, $\left.T_{M}\left(u_{3}, v_{3}\right)\right)$.

Indeed, $\mathscr{T}_{M}(\mathscr{N}(u), \mathscr{N}(v))=\mathscr{T}_{M}\left(\left(u_{3}, 1-u_{2}, u_{1}\right),\left(v_{3}, 1-v_{2}, v_{1}\right)\right)=\left(T_{M}\left(u_{3}, v_{3}\right), S_{M}(1-\right.$ $\left.\left.u_{2}, 1-v_{2}\right), S_{M}\left(u_{1}, v_{1}\right)\right)$, then $\mathscr{N}\left(\mathscr{T}_{M}(\mathscr{N}(u), \mathscr{N}(v))\right)=\left(S_{M}\left(u_{1}, v_{1}\right), 1-S_{M}\left(1-u_{2}, 1-\right.\right.$ $\left.\left.v_{2}\right), T_{M}\left(u_{3}, v_{3}\right)\right)=\left(S_{M}\left(u_{1}, v_{1}\right), T_{M}\left(u_{2}, v_{2}\right), T_{M}\left(u_{3}, v_{3}\right)\right)=\mathscr{S}_{M}(u, v)$. Thus, $\mathscr{T}_{M}$ and $\mathscr{S}_{M}$ are dual with respect to $\mathscr{N}_{s}$.

(2) $\mathscr{T}_{P}(u, v)=\left(T_{P}\left(u_{1}, v_{1}\right), S_{P}\left(u_{2}, v_{2}\right), S_{P}\left(u_{3}, v_{3}\right)\right), \mathscr{S}_{P}(u, v)=\left(S_{P}\left(u_{1}, v_{1}\right), T_{P}\left(u_{2}, v_{2}\right), T_{P}\left(u_{3}, v_{3}\right)\right)$.

(3) $\mathscr{T}(u, v)=\left(T_{L K}\left(u_{1}, v_{1}\right), S_{P}\left(u_{2}, v_{2}\right), S_{P}\left(u_{3}, v_{3}\right)\right), \mathscr{S}(u, v)=\left(S_{P}\left(u_{1}, v_{1}\right), T_{P}\left(u_{2}, v_{2}\right), T_{L K}\left(u_{3}, v_{3}\right)\right)$.

(4) $\mathscr{T}(u, v)=\left(\frac{1}{2}\left(u_{1}+v_{1}-1+u_{1} \cdot v_{1}\right) \vee 0, S_{M}\left(u_{2}, v_{2}\right), S_{M}\left(u_{3}, v_{3}\right)\right), \mathscr{S}(u, v)=\left(S_{M}\left(u_{1}, v_{1}\right)\right.$, $\left.T_{M}\left(u_{2}, v_{2}\right), \frac{1}{2}\left(u_{3}+v_{3}-1+u_{3} \cdot v_{3}\right) \vee 0\right)$.

(5) $\mathscr{T}(u, v)=\left(T_{P}\left(u_{1}, v_{1}\right), S_{M}\left(u_{2}, v_{2}\right), S_{M}\left(u_{3}, v_{3}\right)\right), \mathscr{S}(u, v)=\left(S_{M}\left(u_{1}, v_{1}\right), T_{M}\left(u_{2}, v_{2}\right), T_{P}\left(u_{3}, v_{3}\right)\right)$.

(6) $\mathscr{T}(u, v)=\left(T_{P}\left(u_{1}, v_{1}\right), S_{L K}\left(u_{2}, v_{2}\right), S_{L K}\left(u_{3}, v_{3}\right)\right), \mathscr{S}(u, v)=\left(S_{L K}\left(u_{1}, v_{1}\right), T_{L K}\left(u_{2}, v_{2}\right), T_{P}\left(u_{3}, v_{3}\right)\right)$.

Representable neutrosophic t-norms are mainly analyzed and discussed above. As for non-representable neutrosophic t-norms, we give the following theorem:

Theorem 4. Let $\mathscr{T}:\left(D^{*}\right)^{2} \rightarrow D^{*}$ be a mapping. Then, for all $u, v \in D^{*}$,

$$
\mathscr{T}(u, v)= \begin{cases}u & \text { if } v=1_{D^{*}}, \\ v & \text { if } u=1_{D^{*}} \\ \left(\min \left(u_{1}, v_{1}\right), \max \left(1-u_{1}, 1-v_{1}\right), \max \left(u_{3}, v_{3}\right)\right) & \text { otherwise. }\end{cases}
$$

is a non-representable neutrosophic t-norm.

Proof. Firstly, $\mathscr{T}$ is a neutrosophic t-norm. In fact,

(NT1) Obviously, $\mathscr{T}(u, v)=\mathscr{T}(v, u)$, for all $u, v \in D^{*}$.

(NT2) If $u=1_{D^{*}}$ or $v=1_{D^{*}}$, we can easily prove that $\mathscr{T}(u, \mathscr{T}(v, w))=\mathscr{T}(\mathscr{T}(u, v), w)$. If $u \neq 1_{D^{*}}$ and $v \neq 1_{D^{*}}, \mathscr{T}(u, \mathscr{T}(v, w))=\left(\min \left(u_{1}, \min \left(v_{1}, w_{1}\right)\right), \max \left(1-u_{1}, 1-\min \left(v_{1}, w_{1}\right)\right)\right.$, $\left.\max \left(u_{3}, \max \left(v_{3}, w_{3}\right)\right)\right)=\left(\min \left(u_{1}, v_{1}, w_{1}\right), \max \left(1-u_{1}, 1-v_{1}, 1-w_{1}\right), \max \left(u_{3}, v_{3}, w_{3}\right)\right)=$ $\left(\min \left(\min \left(u_{1}, v_{1}\right), w_{1}\right), \max \left(1-\min \left(u_{1}, v_{1}\right), 1-w_{1}\right), \max \left(\max \left(u_{3}, v_{3}\right), w_{3}\right)\right)=\mathscr{T}(\mathscr{T}(u, v), w)$.

(NT3) $\mathscr{T}\left(1_{D^{*}}, u\right)=u$.

(NT4) If $u=1_{D^{*}}$ or $v=1_{D^{*}}$, we can easily prove that $\mathscr{T}$ is non-decreasing in every variable. If $u \neq 1_{D^{*}}$ and $v \neq 1_{D^{*}}$, let $u, u^{\prime}, v, v^{\prime} \in D^{*}$ with the condition $u \leq_{1} u^{\prime}, v \leq_{1} v^{\prime}$. Then $u_{1} \leq u_{1}^{\prime}, v_{1} \leq v_{1}^{\prime}$, $u_{3} \geq u_{3}^{\prime}, v_{3} \geq v_{3}^{\prime}$. Thus, $\min \left(u_{1}, v_{1}\right) \leq \min \left(u_{1}^{\prime}, v_{1}^{\prime}\right), \max \left(1-u_{1}, 1-v_{1}\right) \geq \max \left(1-u_{1}^{\prime}, 1-v_{1}^{\prime}\right)$, $\max \left(u_{3}, v_{3}\right) \geq \max \left(u_{3}^{\prime}, v_{3}^{\prime}\right)$. That is, $\mathscr{T}(u, v) \leq_{1} \mathscr{T}\left(u^{\prime}, v^{\prime}\right)$. Therefore, $\mathscr{T}$ is a neutrosophic t-norm.

Secondly, for a representable neutrosophic t-norm $\mathscr{T}$, there exists a t-norm $T$ and two t-conorms $S_{1}, S_{2}$ on $[0,1]$ such that, for all $u=\left(u_{1}, u_{2}, u_{3}\right), v=\left(v_{1}, v_{2}, v_{3}\right) \in D^{*}, \mathscr{T}(u, v)=$ $\left(T\left(u_{1}, v_{1}\right), S_{1}\left(u_{2}, v_{2}\right), S_{2}\left(u_{3}, v_{3}\right)\right)$. Let $u=(0.2,0.5,0.7), u^{\prime}=(0.3,0.5,0.7), v=(0.4,0.5,0.9)$. From $\mathscr{T}(u, v)=(0.2,0.8,0.9)$ and $\mathscr{T}\left(u^{\prime}, v\right)=(0.3,0.7,0.9)$, we get $S_{1}\left(u_{2}, v_{2}\right)=0.8$ and $S_{1}\left(u_{2}^{\prime}, v_{2}\right)=0.7$, so $S_{1}\left(u_{2}, v_{2}\right) \neq S_{1}\left(u_{2}^{\prime}, v_{2}\right)$. Hence $S_{1}(u, v)$ is not independent from $u_{1}$, thus $\mathscr{T}$ is not representable.

Furthermore, the dual neutrosophic t-conorm of $\mathscr{T}$ with respect to the standard neutrosophic negator $\mathscr{N}_{s}$ is $\mathscr{S}$ defined by, for all $u, v \in D^{*}$, 


$$
\mathscr{S}(u, v)= \begin{cases}u & \text { if } v=0_{D^{*}} \\ v & \text { if } u=0_{D^{*}} \\ \left(\max \left(u_{1}, v_{1}\right), \min \left(u_{3}, v_{3}\right), \min \left(u_{3}, v_{3}\right)\right) & \text { otherwise. }\end{cases}
$$

Then, $\mathscr{S}$ is not representable.

Remark 1. Let $\mathscr{T}$ be a non-representable neutrosophic t-norm on $D^{*}, \mathscr{S}$ be a neutrosophic t-conorm which is dual to $\mathscr{T}$ with respect to the standard neutrosophic negator $\mathscr{N}_{s}$. Then, $\mathscr{S}$ is not representable. Conversely, the dual neutrosophic t-norm with respect to an involutive neutrosophic negator $\mathscr{N}$ on $D^{*}$ of a non-representable neutrosophic t-conorm is not representable.

Example 6. Let $\mathscr{T}:\left(D^{*}\right)^{2} \rightarrow D^{*}$ be a mapping. Then, for all $u, v \in D^{*}$,

$$
\mathscr{T}(u, v)= \begin{cases}u & \text { if } v=1_{D^{*}}, \\ v & \text { if } u=1_{D^{*}}, \\ \left(\min \left(u_{1}, v_{1}\right), \max \left(1-u_{1}, 1-v_{1}\right), \max \left(1-u_{1}, 1-v_{1}\right)\right) & \text { otherwise. }\end{cases}
$$

is a non-representable neutrosophic t-norm.

Meanwhile, the dual neutrosophic $t$-conorm $\mathscr{S}$ of $\mathscr{T}$ with respect to $\mathscr{N}_{S}$ is presented by, for all $u, v \in D^{*}$,

$$
\mathscr{S}(u, v)= \begin{cases}u & \text { if } v=0_{D^{*}}, \\ v & \text { if } u=0_{D^{*}}, \\ \left(\max \left(1-u_{3}, 1-v_{3}\right), \min \left(u_{3}, v_{3}\right), \min \left(u_{3}, v_{3}\right)\right) & \text { otherwise. }\end{cases}
$$

Then, $\mathscr{S}$ is not representable, too.

Example 7. Let $\mathscr{S}$ be a mapping: $\left(D^{*}\right)^{2} \rightarrow D^{*}$. Then, for all $u, v \in D^{*}$,

$$
\mathscr{S}(u, v)= \begin{cases}u & \text { if } v=0_{D^{*}}, \\ v & \text { if } u=0_{D^{*}} \\ \left(\max \left(1-u_{3}, 1-v_{3}\right), \min \left(u_{2}, v_{2}\right), \min \left(u_{3}, v_{3}\right)\right) & \text { otherwise. }\end{cases}
$$

is a non-representable neutrosophic t-conorm.

Meanwhile, the dual neutrosophic $t$-norm $\mathscr{T}$ of $\mathscr{S}$ with respect to $\mathscr{N}_{s}$ is presented by, for all $u, v \in D^{*}$,

$$
\mathscr{T}(u, v)= \begin{cases}u & \text { if } v=1_{D^{*}}, \\ v & \text { if } u=1_{D^{*}} \\ \left(\min \left(u_{1}, v_{1}\right), \max \left(u_{2}, v_{2}\right), \max \left(1-u_{1}, 1-v_{1}\right)\right) & \text { otherwise. }\end{cases}
$$

Then, $\mathscr{T}$ is not representable, too.

\section{Neutrosophic Residual Implications of Neutrosophic t-Norms}

This section will introduce the notions of neutrosophic residual implications on the complete lattice $D^{*}$, investigate basic properties of neutrosophic residual implications, and give some important conclusions between neutrosophic t-norms and neutrosophic residual implications after proving that residual neutrosophic $t$-norms are $\vee$-distributive. Firstly, we give the notions of neutrosophic implications on $D^{*}$.

Definition 17. A neutrosophic implication is a function $\mathscr{I}:\left(D^{*}\right)^{2} \rightarrow D^{*}$ that satisfies the following conditions,

(NI1) $\mathscr{I}$ is non-increasing with respect to $\leq_{1}$ in its first variable, that is, $\mathscr{I}(u, v) \geq_{1} \mathscr{I}\left(u^{\prime}, v\right)$, where $u, u^{\prime}, v \in D^{*}$ and $u \leq_{1} u^{\prime}$;

(NI2) $\mathscr{I}$ is non-decreasing with respect to $\leq_{1}$ in its second variable, that is, $\mathscr{I}(u, v) \leq_{1} \mathscr{I}\left(u, v^{\prime}\right)$, where $u, v, v^{\prime} \in D^{*}$ and $v \leq_{1} v^{\prime}$

(NI3) $\mathscr{I}\left(0_{D^{*}}, 0_{D^{*}}\right)=1_{D^{*}}$; 
(NI4) $\mathscr{I}\left(1_{D^{*}}, 1_{D^{*}}\right)=1_{D^{*}}$;

(NI5) $\mathscr{I}\left(1_{D^{*}}, 0_{D^{*}}\right)=0_{D^{*}}$.

Definition 18. A function $\mathscr{I}:\left(D^{*}\right)^{2} \rightarrow D^{*}$ is called a neutrosophic residual implication, if there exits a neutrosophic t-norm $\mathscr{T}$ such that

$$
\mathscr{I}(u, v)=\sup \left\{w \mid w \in D^{*}, \mathscr{T}(u, w) \leq_{1} v\right\} .
$$
by $\mathscr{I}_{\mathscr{T}}$.

If $\mathscr{I}$ is a neutrosophic residual implication generated from a neutrosophic t-norm $\mathscr{T}$, then it will be denoted

Furthermore, a neutrosophic t-norm $\mathscr{T}$ satisfies the residual principle if and only if, for all $u, v, w \in D^{*}$,

$$
\mathscr{T}(u, w) \leq_{1} v \text { if and only if } w \leq_{1} \mathscr{I}_{\mathscr{T}}(u, v) .
$$

Similarly, we can get the definitions of neutrosophic co-implications:

Definition 19. A neutrosophic co-implication is a function $\mathscr{J}:\left(D^{*}\right)^{2} \rightarrow D^{*}$ that satisfies the following conditions:

(NJ1) $\mathscr{J}$ is non-increasing with respect to $\leq_{1}$ in its first variable, that is, $\mathscr{J}(u, v) \geq_{1} \mathscr{J}\left(u^{\prime}, v\right)$, where $u, u^{\prime}, v \in D^{*}$ and $u \leq u_{1} u^{\prime}$

(NJ2) $\mathscr{J}$ is non-decreasing with respect to $\leq_{1}$ in its second variable, that is, $\mathscr{J}(u, v) \leq_{1} \mathscr{J}\left(u, v^{\prime}\right)$, where $u, v, v^{\prime} \in D^{*}$ and $v \leq_{1} v^{\prime}$;

(NJ3) $\mathscr{J}\left(0_{D^{*}}, 0_{D^{*}}\right)=0_{D^{*}}$;

(NJ4) $\mathscr{J}\left(1_{D^{*}}, 1_{D^{*}}\right)=0_{D^{*}}$;

(NJ5) $\mathscr{J}\left(0_{D^{*}}, 1_{D^{*}}\right)=1_{D^{*}}$

Definition 20. A function $\mathscr{J}:\left(D^{*}\right)^{2} \rightarrow D^{*}$ is called a neutrosophic residual co-implication, if there exits a neutrosophic t-conorm $\mathscr{S}$ such that

$$
\mathscr{J}(u, v)=\inf \left\{w \mid w \in D^{*}, \mathscr{S}(u, w) \geq_{1} v\right\} .
$$

If $\mathscr{J}$ is a neutrosophic residual co-implication generated from a neutrosophic t-conorm $\mathscr{S}$, then it will be denoted by $\mathscr{J}_{\mathscr{S}}$.

Furthermore, a neutrosophic t-conorm $\mathscr{S}$ satisfies the residual principle if and only if, for all $u, v, w \in D^{*}$,

$$
\mathscr{S}(u, w) \geq_{1} v \text { if and only if } w \geq_{1} \mathscr{I}_{\mathscr{S}}(u, v) .
$$

Using the description of the above definitions, we can easily obtain the neutrosophic residual implications of neutrosophic t-norms discussed in Section 4.

Example 8. The neutrosophic residual implications of the representable neutrosophic t-norms of Example 3 are given by, for all $u, v \in D^{*}$,

(1) $\mathscr{I}_{\mathscr{T}_{M}}(u, v)=\left(I_{G D}\left(u_{1}, v_{1}\right), J_{G D}\left(u_{2}, v_{2}\right), J_{G D}\left(u_{3}, v_{3}\right)\right)$;

(2) $\mathscr{I}_{\mathscr{T}_{P}}(u, v)=\left(I_{G G}\left(u_{1}, v_{1}\right), J_{G G}\left(u_{2}, v_{2}\right), J_{G G}\left(u_{3}, v_{3}\right)\right)$;

(3) $\mathscr{I}_{\mathscr{T}_{L K}}(u, v)=\left(I_{L K}\left(u_{1}, v_{1}\right), J_{L K}\left(u_{2}, v_{2}\right), J_{L K}\left(u_{3}, v_{3}\right)\right)$;

(4) $\mathscr{I}_{\mathscr{T}_{D}}(u, v)=\left(I_{W B}\left(u_{1}, v_{1}\right), J_{W B}\left(u_{2}, v_{2}\right), J_{W B}\left(u_{3}, v_{3}\right)\right)$;

(5) $\mathscr{I}_{\mathscr{T}_{n M}}(u, v)=\left(I_{F D}\left(u_{1}, v_{1}\right), J_{F D}\left(u_{2}, v_{2}\right), J_{F D}\left(u_{3}, v_{3}\right)\right)$;

(6) $\mathscr{I}_{\mathscr{T}}(u, v)=\left(I_{G G}\left(u_{1}, v_{1}\right), J_{L K}\left(u_{2}, v_{2}\right), J_{W B}\left(u_{3}, v_{3}\right)\right)$;

(7) $\mathscr{I}_{\mathscr{T}}(u, v)=\left(I_{W B}\left(u_{1}, v_{1}\right), J_{G G}\left(u_{2}, v_{2}\right), J_{G G}\left(u_{3}, v_{3}\right)\right)$;

(8) $\mathscr{I}_{\mathscr{T}}(u, v)=\left(I_{L K}\left(u_{1}, v_{1}\right), J_{L K}\left(u_{2}, v_{2}\right), J_{G D}\left(u_{3}, v_{3}\right)\right)$; 


$$
\mathscr{I}_{\mathscr{T}}(u, v)=\left(I_{G D}\left(u_{1}, v_{1}\right), J_{G G}\left(u_{2}, v_{2}\right), J_{G D}\left(u_{3}, v_{3}\right)\right)
$$

Example 9. The neutrosophic residual co-implications of the representable neutrosophic t-conorms of Example 4 are given by, for all $u, v \in D^{*}$,

$\begin{array}{ll}\text { (1) } & \mathscr{J}_{\mathscr{S}_{M}}(u, v)=\left(J_{G D}\left(u_{1}, v_{1}\right), I_{G D}\left(u_{2}, v_{2}\right), I_{G D}\left(u_{3}, v_{3}\right)\right) ; \\ \text { (2) } & \mathscr{J}_{\mathscr{S}_{P}}(u, v)=\left(J_{G G}\left(u_{1}, v_{1}\right), I_{G G}\left(u_{2}, v_{2}\right), I_{G G}\left(u_{3}, v_{3}\right)\right) ; \\ (3) & \mathscr{J}_{\mathscr{S}_{L K}}(u, v)=\left(J_{L K}\left(u_{1}, v_{1}\right), I_{L K}\left(u_{2}, v_{2}\right), I_{L K}\left(u_{3}, v_{3}\right)\right) ; \\ (4) & \mathscr{J}_{\mathscr{S}_{D}}(u, v)=\left(J_{W B}\left(u_{1}, v_{1}\right), I_{W B}\left(u_{2}, v_{2}\right), I_{W B}\left(u_{3}, v_{3}\right)\right) ; \\ (5) & \mathscr{I}_{\mathscr{S}_{n M}}(u, v)=\left(J_{F D}\left(u_{1}, v_{1}\right), I_{F D}\left(u_{2}, v_{2}\right), I_{F D}\left(u_{3}, v_{3}\right)\right) ; \\ (6) & \mathscr{J}_{\mathscr{S}}(u, v)=\left(J_{G D}\left(u_{1}, v_{1}\right), I_{G G}\left(u_{2}, v_{2}\right), I_{L K}\left(u_{3}, v_{3}\right)\right) ; \\ (7) & \mathscr{J} \mathscr{S}(u, v)=\left(J_{G G}\left(u_{1}, v_{1}\right), I_{L K}\left(u_{2}, v_{2}\right), I_{L K}\left(u_{3}, v_{3}\right)\right) ; \\ \text { (8) } & \mathscr{J}_{\mathscr{S}}(u, v)=\left(J_{L K}\left(u_{1}, v_{1}\right), I_{L K}\left(u_{2}, v_{2}\right), I_{G D}\left(u_{3}, v_{3}\right)\right) ; \\ (9) & \mathscr{J} \mathscr{S}(u, v)=\left(J_{W B}\left(u_{1}, v_{1}\right), I_{G G}\left(u_{2}, v_{2}\right), I_{F D}\left(u_{3}, v_{3}\right)\right)\end{array}$

As we all know, t-conorms are dual operators of $\mathrm{t}$-norms on $[0,1]$, in the same way, residual co-implications are dual operators of residual implications on $[0,1]$, with respect to $N(u)=1-u$. Neutrosophic residual co-implications of neutrosophic $t$-conorms are dual operators of neutrosophic residual implications of neutrosophic t-norms, just as that neutrosophic t-conorms are dual operators of neutrosophic t-norms with respect to $\mathscr{N}_{s}$. As Examples 8 and 9 above show, if $\mathscr{S}$ is the dual neutrosophic t-conorm of a neutrosophic t-norm $\mathscr{T}$, then the neutrosophic residual co-implication $\mathscr{J}_{\mathscr{S}}$ is the dual operator of the neutrosophic residual implication of $\mathscr{I}_{\mathscr{T}}$.

Next, we will introduce the most important theorem in this section, which gives the sufficient condition that the residual operators induced by neutrosophic t-norms must be neutrosophic implications.

Theorem 5. Let $\mathscr{T}$ be a neutrosophic $t$-norm on $D^{*}$ with the neutral element $1_{D^{*}}$. Then, for all $u, v \in D^{*}$,

$$
\mathscr{I}_{\mathscr{T}}(u, v)=\sup \left\{w \mid w \in D^{*}, \mathscr{T}(u, w) \leq_{1} v\right\}
$$

is a neutrosophic implication.

Proof. From Definition 18, $\mathscr{I}_{\mathscr{T}}\left(u, 1_{D^{*}}\right)=\sup \left\{w \mid w \in D^{*}, \mathscr{T}(u, w) \leq{ }_{1} 1_{D^{*}}\right\}=1_{D^{*}}$, for all $u \in D^{*}$. Therefore, $\mathscr{I}_{\mathscr{T}}\left(1_{D^{*}}, 1_{D^{*}}\right)=1_{D^{*}}$. Since $\mathscr{T}$ is non-decreasing, $\mathscr{I}_{\mathscr{T}}\left(1_{D^{*},} 0_{D^{*}}\right)=\sup \{w \mid w \in$ $\left.D^{*}, \mathscr{T}\left(w, 1_{D^{*}}\right) \leq{ }_{1} 0_{D^{*}}\right\}=\sup \left\{w \mid w \in D^{*}, w \leq_{1} 0_{D^{*}}\right\}=0_{D^{*}} \cdot \mathscr{I}_{\mathscr{T}}\left(0_{D^{*}, 0_{D^{*}}}\right)=\sup \{w \mid w \in$ $\left.D^{*}, \mathscr{T}\left(w, 0_{D^{*}}\right) \leq{ }_{1} 0_{D^{*}}\right\}=1_{D^{*}}$. Let $u, u^{\prime} \in D^{*}$ with the condition $u \leq{ }_{1} u^{\prime}$. Since the non-decreasingness of $\mathscr{T},\left\{w \mid w \in D^{*}, \mathscr{T}(u, w) \leq_{1} v\right\} \supseteq_{1}\left\{w \mid w \in D^{*}, \mathscr{T}\left(u^{\prime}, w\right) \leq_{1} v\right\}$, then $\sup \left\{w \mid w \in D^{*}, \mathscr{T}(u, w) \leq_{1}\right.$ $v\} \geq_{1} \sup \left\{w \mid w \in D^{*}, \mathscr{T}\left(u^{\prime}, w\right) \leq_{1} v\right\}$. Thus, $\mathscr{I}_{\mathscr{T}}(u, v) \geq_{1} \mathscr{I}_{\mathscr{T}}\left(u^{\prime}, v\right)$. That is $\mathscr{I}_{\mathscr{T}}$ is non-increasing with respect to $\leq_{1}$ in its first variable. Let $v, v^{\prime} \in D^{*}$ with the condition $v \leq_{1} v^{\prime}$. Since the non-decreasingness of $\mathscr{T},\left\{w \mid w \in D^{*}, \mathscr{T}(u, w) \leq_{1} v\right\} \subseteq_{1}\left\{w \mid w \in D^{*}, \mathscr{T}(u, w) \leq_{1} v^{\prime}\right\}$, then $\sup \left\{w \mid w \in D^{*}, \mathscr{T}(u, w) \leq_{1} v\right\} \leq_{1} \sup \left\{w \mid w \in D^{*}, \mathscr{T}(u, w) \leq_{1} v^{\prime}\right\}$. Thus, $\mathscr{I}_{\mathscr{T}}(u, v) \leq_{1}$ $\mathscr{I}_{\mathscr{T}}\left(u, v^{\prime}\right)$. That is $\mathscr{I}_{\mathscr{T}}$ is non-decreasing with respect to $\leq_{1}$ in its second variable.

For neutrosophic residual implications, there are several important properties as follows:

Theorem 6. Suppose that $\mathscr{T}$ is a neutrosophic t-norm on $D^{*}$ with the neutral element $1_{D^{*},} \mathscr{I}_{\mathscr{T}}$ is a neutrosophic residual implication. Then, for all $u, v, w \in D^{*}$,

(1) $\mathscr{I}_{\mathscr{T}}\left(0_{\left.D^{*}, v\right)}=1_{D^{*}}\right.$;

(2) $\mathscr{I}_{\mathscr{T}}\left(u, 1_{D^{*}}\right)=1_{D^{*}}$;

(3) $\mathscr{I}_{\mathscr{T}}(u, u)=1_{D^{*}}$;

(4) $\mathscr{I}_{\mathscr{T}}\left(1_{D^{*}}, v\right)=v$;

(5) $\mathscr{I}_{\mathscr{T}}(u, v) \geq{ }_{1} v$; 
(6) $\mathscr{I}_{\mathscr{T}}(u, v)=1_{D^{*}}$ if and only if $u \leq_{1} v$;

(7) $u \leq_{1} \mathscr{I}_{\mathscr{T}}(v, w)$ if and only if $v \leq_{1} \mathscr{I}_{\mathscr{T}}(u, w)$;

(8) $u \leq_{1} \mathscr{I}_{\mathscr{T}}(v, \mathscr{T}(u, v))$;

(9) $\mathscr{I}_{\mathscr{T}}(\mathscr{T}(u, v), \mathscr{T}(u, w)) \geq_{1} \mathscr{I}_{\mathscr{T}}(v, w)$.

Proof. For all $u, v \in D^{*}$,

The proofs of (1)-(4) can be directly obtained by Definition 18 .

(5) Since $\mathscr{I}$ is non-increasing with respect to $\leq_{1}$ in its first variable, $\mathscr{I}_{\mathscr{T}}(u, v) \geq_{1} \mathscr{I}_{\mathscr{T}}\left(1_{D^{*}}, v\right)=v$.

(6) On the one hand, since $u \leq_{1} v, \mathscr{T}\left(1_{D^{*}}, u\right) \leq_{1} v$. Thus, $\mathscr{I}_{\mathscr{T}}(u, v) \geq_{1} 1_{D^{*}}$, that is $\mathscr{I}_{\mathscr{T}}(u, v)=$ $1_{D^{*}}$. On the other hand, if $\mathscr{I}_{\mathscr{T}}(u, v)=1_{D^{*}}$, then $\mathscr{T}\left(1_{D^{*}}, u\right) \leq_{1} v$. Thus, $u \leq_{1} v$.

(7) Since $u \leq_{1} \mathscr{I}_{\mathscr{T}}(v, w), \mathscr{T}(v, u) \leq_{1} w$. Thus, $v \leq_{1} \mathscr{I}_{\mathscr{T}}(u, w)$. Similarly, it follows from $v \leq_{1} \mathscr{I}_{\mathscr{T}}(u, w)$ that $u \leq_{1} \mathscr{I}_{\mathscr{T}}(v, w)$.

(8) Since $\mathscr{T}(u, v) \leq_{1} \mathscr{T}(u, v), u \leq_{1} \mathscr{I}_{\mathscr{T}}(v, \mathscr{T}(u, v))$.

(9) $\mathscr{I}_{\mathscr{T}}(\mathscr{T}(u, v), \mathscr{T}(u, w))=\sup \left\{t \mid t \in D^{*}, \mathscr{T}(\mathscr{T}(u, v), t) \leq_{1} \mathscr{T}(u, w)\right\}=\sup \{t \mid t \in$ $\left.D^{*}, \mathscr{T}(u, \mathscr{T}(v, t)) \leq_{1} \mathscr{T}(u, w)\right\} \geq_{1} \sup \left\{t \mid t \in D^{*}, \mathscr{T}(v, t) \leq_{1} w\right\}=\mathscr{I}_{\mathscr{T}}(v, w)$.

Example 10. Example 8 shows some neutrosophic residual implications of representable neutrosophic $t$-norms, furthermore, it is easy to verify that neutrosophic residual implications of representable neutrosophic $t$-norms satisfy the properties described in Theorem 6.

For non-representable neutrosophic t-norms, take the neutrosophic t-norm $\mathscr{T}$ presented in Theorem 4 for example, then, for all $u, v \in D^{*}$,

$$
\mathscr{I}_{\mathscr{T}}(u, v)= \begin{cases}1_{D^{*}} & \text { if } v=1_{D^{*}}, \\
v & \text { if } u=1_{D^{*}}, \\
\left(I_{G D}\left(u_{1}, v_{1}\right),\left\{\begin{array}{ll}
u_{1} \text { if } u_{1} \leq 1-v_{2}, \\
0 \quad \text { otherwise }
\end{array}, J_{G D}\left(u_{3}, v_{3}\right)\right)\right. & \text { otherwise }\end{cases}
$$

is a neutrosophic implication and satisfies the properties given in Theorem 6.

Similarly, we have the following two important theorems of neutrosophic t-conorm on $D^{*}$ :

Theorem 7. Assume that $\mathscr{S}$ is a neutrosophic t-conorm on $D^{*}$ with the neutral element $0_{D^{*}}$. Then, for all $u, v \in D^{*}$,

$$
\mathscr{J}_{\mathscr{S}}(u, v)=\inf \left\{w \mid w \in D^{*}, \mathscr{S}(u, w) \geq_{1} v\right\} .
$$

is a neutrosophic co-implication.

Proof. From Definition 20, we can prove it using the proven ways of Theorem 5.

Theorem 8. Assume that $\mathscr{S}$ is a neutrosophic t-conorm on $D^{*}$ with the neutral element $0_{D^{*},} \mathscr{J}_{\mathscr{S}}$ is a neutrosophic residual co-implication. Then, for all $u, v, w \in D^{*}$,

(1) $\mathscr{J}_{\mathscr{S}}\left(1_{\left.D^{*}, v\right)}=0_{D^{*}}\right.$;

(2) $\mathscr{J}_{\mathscr{S}}\left(u, 0_{D^{*}}\right)=0_{D^{*}}$;

(3) $\mathscr{J}_{\mathscr{S}}(u, u)=0_{D^{*}}$;

(4) $\mathscr{J}_{\mathscr{S}}\left(0_{D^{*}}, v\right)=v$;

(5) $\mathscr{J}_{\mathscr{S}}(u, v) \leq{ }_{1} v$;

(6) $\mathscr{J}_{\mathscr{S}}(u, v)=0_{D^{*}}$ if and only if $u \geq_{1} v$;

(7) $u \geq_{1} \mathscr{J}_{\mathscr{S}}(v, w)$ if and only if $v \geq_{1} \mathscr{J}_{\mathscr{S}}(u, w)$;

(8) $u \geq_{1} \mathscr{J}_{\mathscr{S}}(v, \mathscr{S}(u, v))$;

(9) $\mathscr{J}_{\mathscr{S}}(\mathscr{S}(u, v), \mathscr{S}(u, w)) \leq_{1} \mathscr{J}_{\mathscr{S}}(v, w)$. 
Example 11. Example 9 shows some neutrosophic residual co-implications of representable neutrosophic t-conorms; furthermore, it is easy to verify that neutrosophic residual co-implications of representable neutrosophic t-conorms satisfy the properties described in Theorem 8.

For non-representable neutrosophic $t$-conorms, take the neutrosophic t-conorm $\mathscr{S}$ presented in Theorem 4 for example, then, for all $u, v \in D^{*}$,

$$
\mathscr{I}_{\mathscr{T}}(u, v)=\left\{\begin{array}{ll}
0_{D^{*}} & \text { if } v=0_{D^{*}}, \\
v & \text { if } u=0_{D^{*}}, \\
\left(J_{G D}\left(u_{1}, v_{1}\right),\left\{\begin{array}{ll}
1 & \text { if } u_{3} \leq v_{2}, \\
v_{2} & \text { otherwise }
\end{array}, I_{G D}\left(u_{3}, v_{3}\right)\right)\right. & \text { otherwise }
\end{array} .\right.
$$

is a neutrosophic co-implication and it satisfies the properties given in Theorem 8.

In Definition $18, \mathscr{I}_{\mathscr{T}}$ is called the neutrosophic residual implication. At the same time, $\mathscr{T}$ is called the residual neutrosophic t-norm. Then, some important properties of the residual neutrosophic t-norm will be discussed below.

Definition 21. [45] A binary operation $H$ on a complete lattice $L$ is called left (right) infinitely $\vee$-distributive, if for all $u \in L$,

$$
H\left(\sup _{w \in W} w, v\right)=\sup _{w \in W} H(w, v)\left(H\left(u, \sup _{w \in W} w\right)=\sup _{w \in W} H(u, w)\right) ;
$$

$H$ is called left (right) infinitely $\wedge$-distributive, if for all $u \in L$,

$$
H\left(\inf _{w \in W} w, v\right)=\inf _{w \in W} H(w, v)\left(H\left(u, \inf _{w \in W} w\right)=\inf _{w \in W} H(u, w)\right),
$$

where $W \subseteq L . H$ is called infinitely $\vee$-distributive ( $\wedge$-distributive) on $L$, if $H$ is both left and right infinitely $\vee$-distributive $(\wedge$-distributive).

Theorem 9. Assume that $\mathscr{T}$ is a residual neutrosophic t-norm on $D^{*}$ with the neutral element $1_{D^{*}}$. Then $\mathscr{T}$ is infinitely $\vee$-distributive on $D^{*}$.

Proof. Let $W \subseteq D^{*}$. If $W=\varnothing$, then $\mathscr{T}\left(\sup _{w \in W} w, v\right)=\mathscr{T}\left(0_{D^{*}}, v\right)=0_{D^{*}}=\sup _{w \in W} \mathscr{T}(w, v)$, for all $v \in D^{*}$. If $W \neq \varnothing$, since $\mathscr{T}$ is non-decreasing, $\mathscr{T}\left(\sup _{w \in W} w, v\right) \geq_{1} \sup _{w \in W} \mathscr{T}(w, v)$, for any $v \in D^{*}$. Suppose $m=\sup _{w \in W} \mathscr{T}(w, v)$, then $\mathscr{T}(w, v) \leq_{1} m$, now we have $w \in\left\{t \mid t \in D^{*}, \mathscr{T}(t, v) \leq_{1} m\right\}$. By Definition $18, w \leq_{1} \mathscr{I}_{\mathscr{T}}(v, m)$, for all $w \in W$. Thus, $\sup _{w} w \leq_{1} \mathscr{I}_{\mathscr{T}}(v, m)$. Since $\mathscr{T}$ is non-decreasing, $\mathscr{T}\left(\sup _{w \in W} w, v\right) \leq_{1} \mathscr{T}\left(\mathscr{I}_{\mathscr{T}}(v, m), v\right)$. Since $\mathscr{T}(u, w) \leq_{1} v$ if and only if $w \leq_{1} \mathscr{I}_{\mathscr{T}}(u, v)$, and $\mathscr{I}_{\mathscr{T}}(v, m) \leq_{1}$ $\mathscr{I}_{\mathscr{T}}(v, m), \mathscr{T}\left(\mathscr{I}_{\mathscr{T}}(v, m), v\right) \leq_{1} m=\sup _{w \in W} \mathscr{T}(w, v)$. Therefore, $\mathscr{T}\left(\sup _{w \in W} w, v\right) \leq_{1} \sup _{w \in W} \mathscr{T}(w, v)$.

Theorem 10. Assume that $\mathscr{T}$ is a residual neutrosophic $t$-norm on $D^{*}$ with the neutral element $1_{D^{*}}$. Then, for all $u, v \in D^{*}$,

(1) $\mathscr{T}\left(\mathscr{I}_{\mathscr{T}}(u, v), u\right) \leq_{1} v$. In particularly, $\mathscr{T}\left(\mathscr{I}_{\mathscr{T}}(u, u), u\right)=u, \mathscr{T}\left(\mathscr{I}_{\mathscr{T}}\left(u, 0_{D^{*}}\right), u\right)=0_{D^{*}} ;$

(2) $\mathscr{I}_{\mathscr{T}}(\mathscr{T}(u, v), w)=\mathscr{I}_{\mathscr{T}}\left(v, \mathscr{I}_{\mathscr{T}}(u, w)\right)$;

(3) $\mathscr{I}_{\mathscr{T}}\left(\sup _{u \in U} u, v\right)=\inf _{u \in U} \mathscr{I}_{\mathscr{T}}(u, v)$;

(4) $\mathscr{T}\left(\mathscr{I}_{\mathscr{T}}(u, v), \mathscr{I}_{\mathscr{T}}(v, w)\right) \leq_{1} \mathscr{I}_{\mathscr{T}}(u, w)$;

(5) $\quad \mathscr{T}\left(\mathscr{I}_{\mathscr{T}}\left(w, 1_{D^{*}}\right), \mathscr{I}_{\mathscr{T}}(u, v)\right) \leq_{1} \mathscr{I}_{\mathscr{T}}(\mathscr{T}(u, w), v)$;

(6) $\mathscr{I}_{\mathscr{T}}\left(u, \mathscr{I}_{\mathscr{T}}(v, w)\right)=\mathscr{I}_{\mathscr{T}}\left(v, \mathscr{I}_{\mathscr{T}}(u, w)\right)$;

(7) $\mathscr{T}\left(\mathscr{I}_{\mathscr{T}}(u, \mathscr{T}(v, u)), u\right)=\mathscr{T}(u, v)$; 


$$
\mathscr{I}_{\mathscr{T}}\left(u, \mathscr{T}\left(\mathscr{I}_{\mathscr{T}}(u, v), u\right)\right)=\mathscr{I}_{\mathscr{T}}(u, v)
$$

Proof. We can directly prove (1)-(6) directly by the method of Theorems 4.3 and 4.6-4.9 in [46]; The proofs of (7) and (8) can be obtained directly from Theorem 3.5 in [48].

Naturally, we can prove that a residual neutrosophic $t$-conorm is infinitely $\wedge$-distributive, and then we can get some important properties of a residual neutrosophic t-conorm on $D^{*}$.

Theorem 11. Assume that $\mathscr{S}$ is a residual neutrosophic t-conorm on $D^{*}$ with the neutral element $0_{D^{*}}$. Then $\mathscr{S}$ is infinitely $\wedge$-distributive on $D^{*}$.

Theorem 12. Assume that $\mathscr{S}$ is a residual neutrosophic t-conorm on $D^{*}$ with the neutral element $0_{D^{*}}$. Then, for all $u, v \in D^{*}$,

(1) $\mathscr{S}\left(\mathscr{J}_{\mathscr{S}}(u, v), u\right) \geq_{1} v$. In particularly, $\mathscr{S}\left(\mathscr{J}_{\mathscr{S}}(u, u), u\right)=u, \mathscr{S}\left(\mathscr{J}_{\mathscr{S}}\left(u, 1_{D^{*}}\right), u\right)=1_{D^{*}} ;$

(2) $\mathscr{J}_{\mathscr{S}}(\mathscr{S}(u, v), w)=\mathscr{J}_{\mathscr{S}}\left(v, \mathscr{J}_{\mathscr{S}}(u, w)\right)$;

(3) $\mathscr{J}_{\mathscr{S}}\left(\inf _{u \in U} u, v\right)=\sup _{u \in U} \mathscr{J}_{\mathscr{S}}(u, v)$;

(4) $\mathscr{S}\left(\mathscr{J}_{\mathscr{S}}(u, v), \mathscr{J}_{\mathscr{S}}(v, w)\right) \geq_{1} \mathscr{J}_{\mathscr{S}}(u, w)$;

(5) $\mathscr{S}\left(\mathscr{J}_{\mathscr{S}}\left(w, 1_{D^{*}}\right), \mathscr{J}_{\mathscr{S}}(u, v)\right) \geq_{1} \mathscr{J}_{\mathscr{S}}(\mathscr{S}(u, w), v)$;

(6) $\mathscr{J}_{\mathscr{S}}\left(u, \mathscr{J}_{\mathscr{S}}(v, w)\right)=\mathscr{J}_{\mathscr{S}}\left(v, \mathscr{J}_{\mathscr{S}}(u, w)\right)$;

(7) $\mathscr{S}\left(\mathscr{J}_{\mathscr{S}}(u, \mathscr{S}(v, u)), u\right)=\mathscr{S}(u, v)$;

(8) $\mathscr{J}_{\mathscr{S}}\left(u, \mathscr{S}\left(\mathscr{J}_{\mathscr{S}}(u, v), u\right)\right)=\mathscr{J}_{\mathscr{S}}(u, v)$.

Proof. The proofs of (1)-(6) can be obtained directly from Theorems 3.2 and 3.5-3.8 in [47]; the proofs of (7) and (8) can be obtained directly from Theorem 3.5 in [48].

\section{Neutrosophic t-Norms Induced by Neutrosophic Implications on $D^{*}$}

From Theorem 5, we know that neutrosophic implications can be induced by neutrosophic t-norms. In this section, the dual situation will be considered. Then, residuated lattices can be constructed on the basis of neutrosophic t-norms and their corresponding neutrosophic residual implications.

Definition 22. Let $\mathscr{I}:\left(D^{*}\right)^{2} \rightarrow D^{*}$ be a neutrosophic implication. The induced operator $\mathscr{T}_{\mathscr{I}}$ by $\mathscr{I}$ is defined as follows:

$$
\mathscr{T}_{\mathscr{I}}(u, v)=\inf \left\{w \mid w \in D^{*}, v \leq_{1} \mathscr{I}(u, w)\right\}, \text { for all } u, v \in D^{*}
$$

Remark 2. (1) $\mathscr{T}_{\mathscr{I}}(u, v)$ is a non-empty set, since $\mathscr{I}\left(u, 1_{D^{*}}\right)=1_{D^{*}}$, for all $u \in D^{*}$. (2) $\mathscr{T}_{\mathscr{I}}$ defined above is not always a neutrosophic $t$-norm. For example, for all $u, v \in D^{*}$,

$$
\mathscr{I}(u, v)=\left(1-u_{1}+u_{1} v_{1},\left\{\begin{array}{ll}
0 & \text { if } u_{2} \geq v_{2}, \\
v_{2} & \text { otherwise }
\end{array},\left\{\begin{array}{ll}
0 & \text { if } u_{3} \geq v_{3 \prime} \prime \\
v_{3} & \text { otherwise }
\end{array}\right)\right.\right.
$$

is a neutrosophic implication. However, $\mathscr{T}_{\mathscr{I}}$ is not a neutrosophic $t$-norm, because $\mathscr{T}_{\mathscr{I}}\left(1_{D^{*}}, v\right)=\left(1, v_{2}, v_{3}\right) \neq v$.

Theorem 13. Let $\mathscr{I}$ be a neutrosophic implication on $D^{*}$. The induced operator $\mathscr{T}_{\mathscr{I}}$ by $\mathscr{I}$ :

$$
\mathscr{T}_{\mathscr{I}}(u, v)=\inf \left\{w \mid w \in D^{*}, v \leq_{1} \mathscr{I}(u, w)\right\}
$$

is a neutrosophic t-norm if $\mathscr{I}$ satisfies the following conditions, for all $u, v, w \in D^{*}$ :

(1) $u \leq_{1} \mathscr{I}(v, w)$ if and only if $v \leq_{1} \mathscr{I}(u, w)$;

(2) $\mathscr{I}(\mathscr{I}(u, v), w)=\mathscr{I}(u, \mathscr{I}(v, w))$;

(3) $\mathscr{I}(u, v)=1_{D^{*}}$ if and only if $u \leq_{1} v$; 
Proof. Firstly, we prove that $\mathscr{T}_{\mathscr{I}}$ is a neutrosophic t-norm.

(NT1) From (1), we can directly get $\mathscr{T}_{\mathscr{I}}(u, v)=\mathscr{T}_{\mathscr{I}}(v, u)$, for all $u, v \in D^{*}$.

(NT2) From (1) and (2), $\mathscr{T}_{\mathscr{I}}\left(\mathscr{T}_{\mathscr{I}}(u, v), w\right)=\mathscr{T}_{\mathscr{I}}\left(w, \mathscr{T}_{\mathscr{I}}(u, v)\right)=\inf \left\{t \mid t \in D^{*}, \mathscr{T}_{\mathscr{I}}(u, v) \leq_{1}\right.$ $\mathscr{I}(w, t)\}=\inf \left\{t \mid t \in D^{*}, u \leq_{1} \mathscr{I}(v, \mathscr{I}(w, t))\right\}=\inf \left\{t \mid t \in D^{*}, v \leq_{1} \mathscr{I}(u, \mathscr{I}(w, t))\right\}=\inf \{t \mid$ $\left.t \in D^{*}, v \leq_{1} \mathscr{I}(w, \mathscr{I}(u, t))\right\}=\inf \left\{t \mid t \in D^{*}, \mathscr{T}_{\mathscr{I}}(v, w) \leq_{1} \mathscr{I}(u, t)\right\}=\mathscr{T}_{\mathscr{I}}\left(\mathscr{T}_{\mathscr{I}}(v, w), u\right)=$ $\mathscr{T}_{\mathscr{I}}\left(u, \mathscr{T}_{\mathscr{I}}(v, w)\right)$.

(NT3) Since $\mathscr{T}_{\mathscr{I}}\left(u, 1_{D^{*}}\right)=\mathscr{T}_{\mathscr{I}}\left(1_{D^{*}}, u\right)=\inf \left\{t \mid t \in D^{*}, u \leq_{1} \mathscr{I}\left(1_{D^{*}}, t\right)\right\}=\inf \left\{t \mid t \in D^{*}, u \leq_{1}\right.$ $t\}=u, \mathscr{T}_{\mathscr{I}}\left(u, 1_{D^{*}}\right)=\mathscr{T}_{\mathscr{I}}\left(1_{D^{*}}, u\right)=u$.

(NT4) Assume $u, u^{\prime}, v, v^{\prime} \in D^{*}$ with the condition $u \leq_{1} u^{\prime}, v \leq_{1} v^{\prime}$. Since $\mathscr{I}$ is a neutrosophic implication, $\mathscr{I}\left(u^{\prime}, t\right) \leq_{1} \mathscr{I}(u, t)$, for all $t \in D^{*}$. For any $t_{0} \in\left\{t \mid t \in D^{*}, v^{\prime} \leq_{1} \mathscr{I}\left(u^{\prime}, t\right)\right\}$, it follows that $v^{\prime} \leq_{1} \mathscr{I}\left(u^{\prime}, t_{0}\right)$. Since $v \leq_{1} v^{\prime}$, and $\mathscr{I}\left(u^{\prime}, t_{0}\right) \leq_{1} \mathscr{I}\left(u, t_{0}\right), v \leq_{1} \mathscr{I}\left(u, t_{0}\right)$, that is, $t_{0} \in\{t \mid$ $\left.t \in D^{*}, v \leq_{1} \mathscr{I}(u, t)\right\}$. Thus, $\left\{t \mid t \in D^{*}, v^{\prime} \leq_{1} \mathscr{I}\left(u^{\prime}, t\right)\right\} \subseteq_{1}\left\{t \mid t \in D^{*}, v \leq_{1} \mathscr{I}(u, t)\right\}$. Hence, $\inf \left\{t \mid t \in D^{*}, v \leq_{1} \mathscr{I}(u, t)\right\} \leq_{1} \inf \left\{t \mid t \in D^{*}, v^{\prime} \leq_{1} \mathscr{I}\left(u^{\prime}, t\right)\right\}$, that is, $\mathscr{T}_{\mathscr{I}}(u, v) \leq_{1} \mathscr{T}_{\mathscr{I}}\left(u^{\prime}, v^{\prime}\right)$.

Therefore, $\mathscr{T}_{\mathscr{I}}$ is a neutrosophic t-norm.

Theorem 13 describes the conditions that an induced operator $\mathscr{T}_{\mathscr{I}}$ by $\mathscr{I}$ is a neutrosophic t-norm. Moreover, we can construct neutrosophic t-norms with neutrosophic implications according to these conditions.

Next, some important properties of the residual neutrosophic implication on $D^{*}$ will be discussed.

Theorem 14. Let $\mathscr{I}$ be a residual neutrosophic implication on $D^{*}$. Then $\mathscr{I}\left(u, \inf _{w \in W} w\right)=\inf _{w \in W} \mathscr{I}(u, w)$, for all $u \in D^{*}, W \subseteq_{1} D^{*}$.

Proof. Let $W \subseteq 1 D^{*}$. If $W=\varnothing$, then $\mathscr{I}\left(u, \inf _{w \in W} w\right)=\mathscr{I}\left(u, 1_{D^{*}}\right)=1_{D^{*}}=\inf _{w \in W} \mathscr{I}(u, w)$, for any $u \in D^{*}$. If $W \neq \varnothing$, since $\mathscr{I}$ is non-decreasingness in its second variable, $\mathscr{I}\left(u, \inf _{w \in W} w\right) \leq_{1} \inf _{w \in W} \mathscr{I}(u, w)$, for all $u \in D^{*}$. Suppose $n=\inf _{w \in W} \mathscr{I}(u, w)$, then $n \leq_{1} \mathscr{I}(u, w)$, now we have $w \in\left\{t \mid t \in D^{*}, n \leq_{1}\right.$ $\mathscr{I}(u, t)\}$. By Definition 22, $\mathscr{T}_{\mathscr{I}}(n, u) \leq_{1} w$, for all $w \in W$. Thus, $\mathscr{T}_{\mathscr{I}}(n, u) \leq_{1} \inf _{w \in W} w$. Since $\mathscr{I}$ is non-decreasingness in its second variable, $\mathscr{I}\left(u, \inf _{w \in W} w\right) \geq_{1} \mathscr{I}\left(u, \mathscr{T}_{\mathscr{I}}(n, u)\right)$. Since $u \leq_{1} \mathscr{I}(v, w)$ if and only if $w \geq_{1} \mathscr{T}_{\mathscr{I}}(u, v)$, and $\mathscr{T}_{\mathscr{I}}(n, u) \geq_{1} \mathscr{T}_{\mathscr{I}}(n, u), \mathscr{I}\left(u, \mathscr{T}_{\mathscr{I}}(n, u)\right) \geq_{1} n=\inf _{w \in W} \mathscr{I}(u, w)$. Therefore, $\mathscr{I}\left(u, \inf _{w \in W} w\right) \geq_{1} \inf _{w \in W} \mathscr{I}(u, w)$.

From Theorem 14, we know that a residual neutrosophic implication satisfies infinitively $\wedge$-distributive in its second variable.

Theorem 15. Assume that $\mathscr{I}$ is a residual neutrosophic implication on $D^{*}$. Then, for all $u, v \in D^{*}$,

(1) $\mathscr{T}_{\mathscr{I}}\left(u, \mathscr{I}\left(u, \mathscr{T}_{\mathscr{I}}(u, v)\right)\right)=\mathscr{T}_{\mathscr{I}}(u, v)$;

(2) $\mathscr{I}\left(u, \mathscr{T}_{\mathscr{I}}(u, \mathscr{I}(u, v))\right)=\mathscr{I}(u, v)$.

Proof. Let $u, v \in D^{*}$.

(1) $\mathscr{T}_{\mathscr{I}}\left(u, \mathscr{I}\left(u, \mathscr{T}_{\mathscr{I}}(u, v)\right)\right)=\inf \left\{t \mid t \in D^{*}, \mathscr{I}\left(u, \inf \left\{t \mid t \in D^{*}, v \leq_{1} \mathscr{I}(u, t)\right\}\right) \leq_{1} \mathscr{I}(u, t)\right\}=$ $\inf \left\{t \mid t \in D^{*}, \inf \left\{\mathscr{I}(u, t) \mid t \in D^{*}, v \leq_{1} \mathscr{I}(u, t)\right\} \leq_{1} \mathscr{I}(u, t)\right\}=\inf \left\{t \mid t \in D^{*}, v \leq_{1}\right.$ $\mathscr{I}(u, t)\}=\mathscr{T}_{\mathscr{I}}(u, v)$.

(2) $\mathscr{I}\left(u, \mathscr{T}_{u, \mathscr{I}}(\mathscr{I}(u, v))\right)=\mathscr{I}\left(u, \inf \left\{t \mid t \in D^{*}, \mathscr{I}(u, v) \leq_{1} \mathscr{I}(u, t)\right\}\right)=\inf \{\mathscr{I}(u, t) \mid t \in$ $\left.D^{*}, \mathscr{I}(u, t) \geq_{1} \mathscr{I}(u, v)\right\}=\mathscr{I}(u, v)$.

Summarizing the results in Theorem 5 and 13, we get the following theorem. 
Theorem 16. (1) Assume that $\mathscr{T}$ is a neutrosophic t-norm on $D^{*}$. Then $\mathscr{I}_{\mathscr{T}}\left(u, \inf _{v \in V} v\right)=\inf _{v \in V} \mathscr{I}_{\mathscr{T}}(u, v)$ and $\mathscr{T}=\mathscr{T}_{\mathscr{I}_{\mathscr{T}}} ;$

(2) Let $\mathscr{I}$ be a neutrosophic implication on $D^{*}$. Then $\mathscr{T}_{\mathscr{I}}$ which satisfies the conditions presented in Theorem 13 is a infinitely $\vee$-distributive neutrosophic t-norm, and $\mathscr{I}=\mathscr{I}_{\mathscr{T}_{\mathscr{I}}}$.

Proof. (1) From Theorem 5, $\mathscr{I}_{\mathscr{T}}$ is a neutrosophic implication. Next, we prove $\mathscr{I}_{\mathscr{T}}\left(u, \inf _{v \in V} v\right)=$ $\inf _{v \in V} \mathscr{I}_{\mathscr{T}}(u, v)$, for all $u \in D^{*}, V \subseteq_{1} D^{*}$. Suppose $V \subseteq_{1} D^{*}$. If $V=\varnothing$, then $\mathscr{I}_{\mathscr{T}}\left(u, \inf _{v \in V} v\right)=$ $\mathscr{I}_{\mathscr{T}}\left(u, 1_{D^{*}}\right)=1_{D^{*}}=\inf _{v \in V} \mathscr{I}_{\mathscr{T}}(u, v)$, for all $u \in D^{*}$. If $V \neq \varnothing$, then $\mathscr{I}_{\mathscr{T}}\left(u, \inf _{v \in V} v\right)=\sup \{t \mid t \in$ $\left.D^{*}, \mathscr{T}(t, u) \leq_{1} \inf _{v \in V} v\right\}=\sup \left\{t \mid t \in D^{*}, \forall v \in V, \mathscr{T}(t, u) \leq_{1} v\right\}=\sup \left\{t \in D^{*} \mid \forall v \in V, t \leq_{1}\right.$ $\left.\mathscr{I}_{\mathscr{T}}(u, v)\right\}=\sup \left\{t \in D^{*} \mid t \leq \inf _{v \in V} \mathscr{I}_{\mathscr{T}}(u, v)\right\}=\inf _{v \in V} \mathscr{I}_{\mathscr{T}}(u, v)$. Finally, from Definitions 18 and 22, we get $\mathscr{T}_{\mathscr{I}}(u, v)=\inf \left\{t \mid t \in D^{*}, v \leq_{1} \mathscr{I}_{\mathscr{T}}(u, t)\right\}=\inf \left\{t \mid t \in D^{*}, \mathscr{T}(u, v) \leq_{1} t\right\}=\mathscr{T}(u, v)$, for all $u, v \in D^{*}$. Thus, $\mathscr{T}=\mathscr{T}_{\mathscr{T}}$.

(2) From Definition 22 and Theorem 13, $\mathscr{T}_{\mathscr{I}}$ is a neutrosophic t-norm. Next, we prove $\mathscr{T}_{\mathscr{I}}\left(\sup _{u \in U} u, v\right)=$ $\sup _{u \in U} \mathscr{T}_{\mathscr{I}}(u, v)$, for all $v \in D^{*}, U \subseteq_{1} D^{*}$. Suppose $U \subseteq_{1} D^{*}$. If $U=\varnothing$, then $\mathscr{T}_{\mathscr{I}}(\sup u, v)=$ $\mathscr{T}_{\mathscr{I}}\left(0_{D^{*}}, v\right)=0_{D^{*}}=\sup _{\mathscr{T}} \mathscr{T}_{\mathscr{I}}(u, v)$, for all $v \in D^{*}$. If $U \neq \varnothing$, then $\mathscr{T}_{\mathscr{I}}(\sup u, v)=\inf \{t \mid t \in$ $\left.D^{*}, v \leq_{1} \mathscr{I}\left(\sup _{u \in U} u, t\right)\right\} \stackrel{u \in U}{=} \inf \left\{t \mid t \in D^{*}, \forall u \in U, v \leq_{1} \mathscr{I}(u, t)\right\}=\inf \left\{\left.t\right|^{u \in U} t \in D^{*}, \forall u \in U, t \geq 1\right.$ $\left.\mathscr{T}_{\mathscr{I}}(u, v)\right\}=\inf \left\{t \mid t \in D^{*}, t \geq \sup _{u \in U} \mathscr{T}_{\mathscr{I}}(u, v)\right\}=\sup _{u \in U} \mathscr{T}_{\mathscr{I}}(u, v)$. Since $\mathscr{T}_{\mathscr{I}}$ satisfies the commutative law, $\mathscr{T}_{\mathscr{I}}\left(u, \sup _{v \in V} v\right)=\sup _{v \in V} \mathscr{T}_{\mathscr{I}}(u, v)$. Hence, $\mathscr{T}_{\mathscr{I}}$ is infinitely $\vee$-distributive. At last, from Definitions 18 and 22, we get $\mathscr{I}_{\mathscr{T}_{\mathscr{I}}}(u, v)=\sup \left\{t \mid t \in D^{*}, \mathscr{T}_{\mathscr{I}}(t, u) \leq_{1} v\right\}=\sup \left\{t \mid t \in D^{*}, t \leq_{1} \mathscr{I}(u, v)\right\}=$ $\mathscr{I}(u, v)$, for all $u, v \in D^{*}$. Thus, $\mathscr{I}=\mathscr{I}_{\mathscr{T}_{\mathscr{I}}}$.

Sections 4 and 5 mainly discuss neutrosophic t-norms and their residual implications, then, we can get a residuated lattice by using these two neutrosophic logic operators as follows:

Theorem 17. Let $\mathscr{T}$ be a neutrosophic t-norm on $D^{*}$. Suppose $\left(D^{*} ; \vee_{1}, \wedge_{1},{ }^{c}, 0_{D^{*}}, 1_{D^{*}}\right)$ is a system on $D^{*}$. For all $u, v \in D^{*}$, we define:

$$
u \otimes v=\mathscr{T}_{\mathscr{I}_{\mathscr{T}}}(u, v) ; u \rightarrow v=\mathscr{I}_{\mathscr{T}}(u, v)
$$

Then, $\left(D^{*} ; \vee_{1}, \wedge_{1}, \otimes, \rightarrow, 0_{D^{*}}, 1_{D^{*}}\right)$ is a residuated lattice.

Proof. Firstly, from Proposition 3, we know that $\left(D^{*} ; \vee_{1}, \wedge_{1}, 0_{D^{*}}, 1_{D^{*}}\right)$ is a bounded lattice.

Then, we prove that $\left(D^{*} ; \otimes, 1_{D^{*}}\right)$ is a commutative monoid. (1) For any $u \in D^{*}, 1_{D^{*}} \otimes u=\inf \{t \mid$ $\left.t \in D^{*}, 1_{D^{*}} \leq_{1} \mathscr{I}_{\mathscr{T}}(u, t)\right\}=\inf \left\{t \mid t \in D^{*}, \mathscr{I}_{\mathscr{T}}(u, t)=1_{D^{*}}\right\}=\inf \left\{t \mid t \in D^{*}, u \leq{ }_{1} t\right\}=u$, $u \otimes 1_{D^{*}}=\inf \left\{t \mid t \in D^{*}, u \leq_{1} \mathscr{I}_{\mathscr{T}}\left(1_{D^{*}}, t\right)\right\}=\inf \left\{t \mid t \in D^{*}, u \leq_{1} t\right\}=u$. Thus, $1_{D^{*}} \otimes u=u \otimes 1_{D^{*}}=u$. (2) Theorem 16 proves that $\mathscr{T}_{\mathscr{I}}=\mathscr{T}$ is a neutrosophic t-norm. Thus, $\mathscr{T}$ satisfies the commutative law, that is, $u \otimes v=v \otimes u$. (3) Similarly, $\mathscr{T}$ satisfies the associative law, that is, $u \otimes(v \otimes w)=(u \otimes v) \otimes w$.

Finally, we prove that $\otimes$ is a binary operation for which the equivalence

$$
u \otimes v \leq_{1} w \text { if and only if } v \leq_{1} u \rightarrow w
$$

holds for all $u, v, w \in D^{*}$. On the one hand, by the definition of $\otimes$, we have $u \otimes v=\inf \{w \mid w \in$ $\left.D^{*}, v \leq_{1} u \rightarrow w\right\}$, then $u \otimes v \leq_{1} w$. Thus, $v \leq_{1} u \rightarrow w$. On the other hand, from the definition of $\rightarrow$, we have $u \rightarrow w=\sup \left\{v \mid v \in D^{*}, u \otimes v \leq_{1} w\right\}$. Thus, $u \otimes v \leq_{1} w$.

Therefore, $\left(D^{*} ; \vee_{1}, \wedge_{1}, \otimes, \rightarrow, 0_{D^{*}}, 1_{D^{*}}\right)$ is a residuated lattice.

Example 12. Suppose $\left(D^{*} ; \vee_{1}, \wedge_{1}{ }^{c}, 0_{D^{*}}, 1_{D^{*}}\right)$ is a system on $D^{*}$. For all $u, v \in D^{*}$, we define: 


$$
\begin{gathered}
u \otimes v=\mathscr{T}_{\mathscr{I}_{\mathscr{T}}}(u, v) \\
u \rightarrow v=\mathscr{I}_{\mathscr{T}}(u, v) .
\end{gathered}
$$

where $\mathscr{T}_{\mathscr{I}_{\mathscr{T}}}(u, v)$ is that presented in Theorem $4, \mathscr{I}_{\mathscr{T}}(u, v)$ is that presented in Example 10.

Then, $\left(D^{*} ; \vee_{1}, \wedge_{1}, \otimes, \rightarrow, 0_{D^{*}}, 1_{D^{*}}\right)$ is a residuated lattice.

Proof. Firstly, from Proposition 3 , we know that $\left(D^{*} ; \vee_{1}, \wedge_{1}, 0_{D^{*}}, 1_{D^{*}}\right)$ is a bounded lattice.

Then, we prove that $\left(D^{*} ; \otimes, 1_{D^{*}}\right)$ is a commutative monoid. (1) For any $u \in D^{*}$, by the definition of $\otimes$, we get $1_{D^{*}} \otimes u=u \otimes 1_{D^{*}}=u$. (2) Obviously, $u \otimes v=v \otimes u$. (3) Suppose $u, v, w \in$ $D^{*}$. If at least one of them is equal to $1_{D^{*}}$, then $u \otimes(v \otimes w)=(u \otimes v) \otimes w$. Otherwise, $u \otimes(v \otimes w)=\left(\min \left(u_{1}, \min \left(v_{1}, w_{1}\right)\right), \max \left(1-u_{1}, \max \left(1-v_{1}, 1-w_{1}\right)\right), \max \left(u_{3}, \max \left(v_{3}, w_{3}\right)\right)\right)=$ $\left(\min \left(u_{1}, v_{1}, w_{1}\right), \max \left(1-u_{1}, 1-v_{1}, 1-w_{1}\right), \max \left(u_{3}, v_{3}, w_{3}\right)\right)=\left(\min \left(v_{1}, \min \left(u_{1}, w_{1}\right)\right), \max (1-\right.$ $\left.\left.v_{1}, \max \left(1-u_{1}, 1-w_{1}\right)\right), \max \left(v_{3}, \max \left(u_{3}, w_{3}\right)\right)\right)=(u \otimes v) \otimes w$.

Finally, we will prove

$$
u \otimes v \leq_{1} w \Leftrightarrow v \leq_{1} u \rightarrow w, \text { for all } u, v, w \in D^{*} .
$$

If $u=1_{D^{*}}, u \otimes v=v \leq_{1} w$ if and only if $v \leq_{1} u \rightarrow w \Rightarrow v \leq_{1} 1_{D^{*}} \rightarrow w \Rightarrow v \leq_{1} w$; If $v=1_{D^{*}}$, $u \otimes v=u \otimes 1_{D^{*}}=u \leq_{1} w \Rightarrow u \leq_{1} 1_{D^{*}}$ if and only if $v \leq_{1} u \rightarrow w \Rightarrow 1_{D^{*}} \leq 1_{D^{*}} ;$ If $u \neq 1_{D^{*}}$ and $v \neq$ $1_{D^{*}}, u \otimes v=\left(\min \left(u_{1}, \min \left(v_{1}, w_{1}\right)\right), \max \left(1-u_{1}, \max \left(1-v_{1}, 1-w_{1}\right)\right), \max \left(u_{3}, \max \left(v_{3}, w_{3}\right)\right)\right) \leq_{1} w$ if and only if

$$
v \leq_{1} \begin{cases}1_{D^{*}} & \text { if } w=1_{D^{*}} \\
w & \text { if } u=1_{D^{*}}, \\
\left(I_{G D}\left(u_{1}, w_{1}\right),\left\{\begin{array}{ll}
u_{1} & \text { if } u_{1} \leq 1-w_{2}, \\
0 & \text { otherwise }
\end{array} J_{G D}\left(u_{3}, w_{3}\right)\right)\right. & \text { otherwise }\end{cases}
$$

that is, $v \leq_{1} u \rightarrow w$.

Therefore, $\left(D^{*} ; \vee_{1}, \wedge_{1}, \otimes, \rightarrow, 0_{D^{*}}, 1_{D^{*}}\right)$ is a residuated lattice.

\section{Conclusions}

Neutrosophic logic plays a vital role in neutrosophic set theory. Neutrosophic t-norms, t-conorms, negators and implications are very important neutrosophic logic operators. In this paper, under the first type inclusion relation, the lattice structure of neutrosophic sets is discussed, $\left(D^{*} ; \leq_{1}\right)$ and $\left(D^{*} ; \vee_{1}, \wedge_{1}{ }^{c}, 0_{D^{*}}, 1_{D^{*}}\right)$ are proved to be a complete lattice and De Morgan algebra, respectively. On the complete lattice $\left(D^{*} ; \leq_{1}\right)$, we introduce the definitions of neutrosophic t-norms, $\mathrm{t}$-conorms, negators and their operations. Furthermore, De Morgan neutrosophic triples are defined, which describe that neutrosophic t-norms and t-conorms are dual with respect to the standard neutrosophic negator. Then, we introduce neutrosophic residual implications (co-implications) on the complete lattice $\left(D^{*} ; \leq_{1}\right)$, propose a theorem which shows that residual operations induced by neutrosophic t-norms are neutrosophic implications, investigate basic properties for neutrosophic residual implications (co-implications), prove that residual neutrosophic t-norms are infinitely $\vee$-distributive, and give some important results for residual neutrosophic t-norms and neutrosophic residual implications. Finally, we introduce neutrosophic operations produced by neutrosophic implications, discuss the conditions that the neutrosophic operations are neutrosophic t-norms, and then construct residuated lattices. Based on these results, we will consider their applications in neutrosophic inference systems in the future.

Author Contributions: This paper is written through contributions of all authors. The individual contributions and responsibilities of all authors can be described as follows: The idea of this whole thesis was put forward by X.Z., he also provided valuable and helpful suggestions throughout the writing of the article. Q.H. investigated the work and completed the paper.

Funding: This work was supported by the National Natural Science Foundation of China (Grant no. 61573240).

Conflicts of Interest: The authors declare no conflict of interest. 


\section{References}

1. Smarandache, F. Neutrosophy: Neutrosophic Probability, Set, and Logic: Analytic Synthesis and Synthetic Analysis; American Research Press: Santa Fe, NM, USA, 1998; pp. 25-38.

2. Wang, H.; Smarandache, F.; Zhang, Y.; Sunderraman, R. Single valued neutrosophic sets. Multispace Multistruct. Neutrosophic Transdiscipl. 2010, 4, 410-413.

3. Ye, J.; Smarandache, F. Similarity measure of refined single-valued neutrosophic sets and its multicriteria decision making method. Neutrosophic Sets Syst. 2016, 12, 41-44.

4. Ye, J. Vector similarity measures of simplified neutrosophic sets and their application in multicriteria decision making. Int. J. Fuzzy Syst. 2014, 16, 204-211.

5. Liu, P. The aggregation operators based on archimedean t-conorm and t-norm for single-valued neutrosophic numbers and their application to decision making. Int. J. Fuzzy Syst. 2016, 18, 849-863. [CrossRef]

6. Ye, J. Multicriteria decision-making method using the correlation coefficient under single-valued neutrosophic environment. Int. J. Gen. Syst. 2013, 42, 386-394. [CrossRef]

7. Hu, Q.; Zhang, X. New similarity measures of single-valued neutrosophic multisets based on the decomposition theorem and its application in medical diagnosis. Symmetry 2018, 10, 466. [CrossRef]

8. Wang, J.; Zhang, X. Two types of single-valued neutrosophic covering rough sets and an application to decision making. Symmetry 2018, 10, 710. [CrossRef]

9. Zhang, X.; Mao, X.; Wu, Y.; Zhai, X. Neutrosophic filters in pseudo-BCI algebras. Int. J. Uncertain Quantif. 2018, 8, 511-526. [CrossRef]

10. Zhang, X.; Hu, Q.; Smarandache, F.; An, X. On neutrosophic triplet groups: Basic properties, NT-subgroups and some notes. Symmetry 2018, 10, 289. [CrossRef]

11. Ma, Y.; Zhang, X.; Yang, X.; Zhou, X. Generalized neutrosophic extended triplet group. Symmetry 2019, 11, 327. [CrossRef]

12. Wu, X.; Zhang, X. The decomposition theorems of AG-neutrosophic extended triplet loops and strong AG-(1, 1)-loops. Mathematics 2019, 7, 268. [CrossRef]

13. Cuong, B.C.; Kreinovich, V. Picture fuzzy sets-a new concept for computational intelligence problems. In Proceedings of the IEEE Third World Congress on Information and Communication Technologies, Hanoi, Vietnam, 15-18 December 2013; pp. 1-6.

14. Zhang, X.; Bo, C.; Smarandache, F.; Dai, J. New inclusion relation of neutrosophic sets with applications and related lattice structure. Int. J. Mach. Learn. Cybern. 2018, 9, 1753-1763. [CrossRef]

15. Klement, E.P.; Mesiar, R.; Stupn̆anová, A. Picture fuzzy sets and 3-fuzzy sets. In Proceedings of the IEEE International Conference on Fuzzy Systems, Rio de Janeiro, Brazil, 8-13 July 2018.

16. Cuong, B.C.; Phong, P.H.; Smarandache, F. Standard neutrosophic soft theory: Some first results. Neutrosophic Sets Syst. 2016, 12, 80-91.

17. Cuong, B.C.; Ngan, R.T.; Ngoc, L.C. Some Algebraic Properties of Picture Fuzzy t-Norms and Picture Fuzzy t—Conorms on Standard Neutrosophic Sets. 2017. Available online: http:/ / vixra.org/abs/1701.0144 (accessed on 20 May 2019).

18. Smarandache, F. Neutrosophic set-A generialization of the intuitionistics fuzzy sets. J. Def. Manag. 2006, 1, 38-42.

19. Smarandache, F. A unifying field in logics: Neutrosophic logic. Multiple-Valued Log. 2002, 8, 385-438.

20. Menger, K. Statistical metrics. Natl. Acad. Sci. 1942, 28, 535-537. [CrossRef]

21. Schweizer, B.; Sklar, A. Associative functions and statistical triangle inequalities. Publ. Math. 1961, 8, 169-186.

22. Schweizer, B.; Sklar, A. Probabilistic Metric Spaces; North-Holland: New York, NY, USA, 1983.

23. Simon, D. Fuzzy sets and fuzzy logic: Theory and applications. Control Eng. Pract. 1996, 4, $1332-1333$. [CrossRef]

24. Klement, E.P.; Mesiar, R.; Pap, E. Triangular norms. Position paper I: Basic analytical and algebraic properties. Fuzzy Sets Syst. 2004, 143, 5-26. [CrossRef]

25. Li, D. Type-2 triangular norms and their residual operators. Inf. Sci. 2015, 317, 259-277. [CrossRef]

26. Zhang, B. Notes on type-2 triangular norms and their residual operators. Inf. Sci. 2016, 346-347, 338-350. [CrossRef]

27. Baczyński, M.; Jayaram, B. Fuzzy implications. Stud. Fuzziness Soft Comput. 2008, 231, 41-105.

28. Zhang, X.; Borzooei, R.A.; Jun, Y.B. Q-filters of quantum B-algebras and basic implication algebras. Symmetry 2018, 10, 573. [CrossRef] 
29. Hu, B.; Kwong, C.K. On type-2 fuzzy sets and their t-norm operations. Inf. Sci. 2014, 255, 58-81. [CrossRef]

30. Wang, C.; Hu, B. On fuzzy-valued operations and fuzzy-valued fuzzy sets. Fuzzy Sets Syst. 2015, 268, 72-92. [CrossRef]

31. Fodor, J.C. Contrapositive symmetry of fuzzy implications. Fuzzy Sets Syst. 1995, 69, 141-156. [CrossRef]

32. Klement, E.P., Navara, M. A survey on different triangular norm-based fuzzy logics. Fuzzy Sets Syst. 1999, 101, 241-251. [CrossRef]

33. Zhou, H. Probabilistically Quantitative Logic and Its Application; Science Press: Beijing, China, 2015; pp. $219-235$.

34. Deschrijver, G. Implication functions in interval-valued fuzzy set theory. Stud. Fuzziness Soft Comput. 2013, 300, 73-99.

35. Deschrijver, G.; Cornelis, C.; Kerre, E.E. On the representation of intuitionistic fuzzy t-norms and t-conorms. IEEE Trans. Fuzzy Syst. 2004, 12, 45-61. [CrossRef]

36. Deschrijver, G.; Kerre, E.E. Classes of intuitionistic fuzzy t-norms satisfying the residuation principle. Int. J. Univ. Fuzziness Knowl.-Based Syst. 2003, 11, 691-709. [CrossRef]

37. Goguen, J.A. L-fuzzy sets. J. Math. Anal. Appl. 1967, 18, 145-174. [CrossRef]

38. Cuong, B.C. Picture fuzzy sets. J. Comput. Sci. Cybern. 2014, 30, 409-420.

39. Cuong, B.C.; Hai, P.V. Some fuzzy logic operators for picture fuzzy sets. In Proceedings of the IEEE Seventh International Conference on Knowledge and Systems Engineering, Ho Chi Minh City, Vietnam, 8-10 October 2015; pp. 132-137.

40. Cuong, B.C.; Kreinovich, V.; Ngan, R.T. A classification of representable t-norm operators for picture fuzzy sets. In Proceedings of the IEEE Eighth International Conference on Knowledge and Systems Engineering (KSE), Hanoi, Vietnam, 6-8 October 2016; pp. 19-24.

41. Cuong, B.C.; Ngan, R.T.; Hai, B.D. An involutive picture fuzzy negator on picture fuzzy sets and some De Morgan triples. In Proceedings of the IEEE Seventh International Conference on Knowledge and Systems Engineering (KSE), Ho Chi Minh City, Vietnam, 8-10 October 2015; pp. 126-131.

42. Cuong, B.C.; Ngan, R.T.; Long, L.B. Some new De Morgan picture operator triples in picture fuzzy logic. J. Comput. Sci. Cybern. 2017, 33, 143-164.

43. Son, L.H.; Viet, P.V.; Hai, P.V. Picture inference system: A new fuzzy inference system on picture fuzzy set. Appl. Intell. 2016, 46, 1-18. [CrossRef]

44. Baets, B.D.; Fodor, J. Residual operators of uninorms. Soft Comput. 1999, 3, 89-100. [CrossRef]

45. Wang, Z.; Yu, Y. Pseudo-t-norms and implication operators on a complete Brouwerian lattice. Fuzzy Sets Syst. 2002, 132, 113-124. [CrossRef]

46. Wang, Z.; Fang, J. Residual operations of left and right uninorms on a complete lattice. Fuzzy Sets Syst. 2009, 160, 22-31. [CrossRef]

47. Wang, Z.; Fang, J. Residual coimplicators of left and right uninorms on a complete lattice. Fuzzy Sets Syst. 2008, 160, 2086-2096. [CrossRef]

48. Liu, H. Semi-uninorms and implications on a complete lattice. Fuzzy Sets Syst. 2012, 191, 72-82. [CrossRef]

49. Alkhazaleh, S. More on neutrosophic norms and conforms. Neutrosophic Sets Syst. 2015, 9, 23-30.

50. Smarandache, F. N-valued refined neutrosophic logic and its applications to physics. Neutrosophic Theory Appl. Progress Phys. 2013, 4, 36-44.

51. Smarandache, F. N-norm and n-conorm in neutrosophic logic and set, and the neutrosophic topologies. In A Unifying Field in Logics: Neutrosophic Logic. Neutrosophy, Neutrosophic Set, Neutrosophic Probability, 4th ed.; American Research Press: Santa Fe, NM, USA, 2005.

52. Atanassov, K. Intuitionistic fuzzy sets. Fuzzy Sets Syst. 1986, 20, 87-96. [CrossRef]

53. Smarandache, F. Neutrosophic Perspectives: Triplets, Duplets, Multisets, Hybrid Operators, Modal Logic, Hedge Algebras. And Applications; Infinite Study: Conshohocken, PA, USA, 2017.

54. Zhang, X.; Bo, C.; Smarandache, F.; Park, C. New operations of tatally dependent-neutrosophic sets and tatally dependent-neutrosophic soft sets. Symmetry 2018, 10, 187. [CrossRef]

55. Sevcovic, D. Free non-distributive morgan-stone algebras. N. Z. J. Math. 1996, 25, 85-94.

(C) 2019 by the authors. Licensee MDPI, Basel, Switzerland. This article is an open access article distributed under the terms and conditions of the Creative Commons Attribution (CC BY) license (http:/ / creativecommons.org/licenses/by/4.0/). 